\title{
The evolution of near infrared spectroscopy in urology
}

\author{
Andrew J. Macnab a,b,c,* \\ ${ }^{a}$ Department of Pediatrics and Associate Member Department of Urology, Faculty of Medicine, \\ University of British Columbia, Vancouver, BC, Canada \\ ${ }^{\mathrm{b}}$ NIRS Study Group, Bladder Care Centre, UBC Hospital, Vancouver, BC, Canada \\ ${ }^{c}$ Stellenbosch Institute for Advanced Study, Wallenberg Research Centre, Stellenbosch, South Africa
}

\begin{abstract}
In the 50 year history of NIRS, biomedical applications in urology are recent. This review summarizes technical advances and novel enquiry that have led to hemodynamic monitoring of the testis, measures of renal function, evaluation of the pelvic floor in women, and interrogation of the bladder. An overview is given of how bladder spectroscopy has evolved and the evidence that non-invasive transcutaneous optical monitoring of the anterior bladder wall now enables variations in the organ's hemodynamics and oxygen supply and demand to be identified in real time. Research indicates that bladder blood flow and oxygenation differ in health and disease, and because NIRS monitors changes in the microcirculation bladder spectroscopy adds physiologic information not available by other means, including via the current invasive method for evaluation of voiding dysfunction.

Confidence that bladder NIRS yields physiologic data comes from the patterns of chromophore change and tissue oxygen saturation observed corresponding to variations in these parameters in NIRS studies of other tissues in response to known physiologic events. Importantly, when interpreted based on this prior research, novel insight is gained about probable causal pathologies underlying bladder dysfunction. Studies to date warrant further research, continued refinement of instrumentation, extension of the scope of monitoring, and exploration of diagnostic software algorithms.
\end{abstract}

Keywords: Bladder, near infrared spectroscopy (NIRS), spatially resolved spectroscopy (SRS), urinary incontinence, urinary tract, urology
Abbreviations
CAD computer assisted design;
CART classification and regression tree;
$\mathrm{CCO}$ cytochrome $\mathrm{C}$ oxidase;
CDU color Doppler ultrasound;
CSE computer systems engineering;
$\mathrm{CW}$ continuous wave;
BOO bladder outlet obstruction;
DO detrusor overactivity;
DPS differential path length spectroscopy;

\footnotetext{
*Address for correspondence: Andrew J. Macnab, Room 324, BC Children's and Women's Hospital, 4500 Oak Street, Vancouver, BC, Canada V6H 3N1. Tel.: +1 604875 2850; Fax: +1 604875 2530; E-mail: amacnab@cw.bc.ca.
} 
fNIRS functional near infrared spectroscopy;

FS fluorescence spectroscopy;

$\mathrm{HHb}$ deoxygenated hemoglobin;

HRUS high resolution ultrasound;

IOD inter-optode distance;

LEDs light emitting diodes;

NIR near infrared;

NIRS near infrared spectroscopy;

$\mathrm{OAB}$ overactive bladder;

$\mathrm{O}_{2} \mathrm{Hb}$ oxygenated hemoglobin;

PFM pelvic floor muscles;

RS Raman spectroscopy;

$\mathrm{SaO}_{2}$ oxygen saturation;

SCI spinal cord injury;

SRS spatially resolved spectroscopy;

$\mathrm{tHb}$ total hemoglobin;

UDS urodynamic pressure flow studies.

\section{Introduction}

Over the last 50 years near infrared spectroscopy (NIRS) has come to be used in a wide range of scientific fields, including in vivo biomedical applications where since 1977 near infrared (NIR) light in the 700-1000 nm wavelength range has been used to study changes in the concentration of hemoglobin in the microcirculation $[44,59,142]$. Applications of NIRS in the field of urology are recent; innovation has led to studies of the testes, organs and structures in the urinary tract including the bladder, and the muscles of the pelvic floor [72,125]. Transcutaneous monitoring of the bladder as it fills and empties is a novel use of NIRS in a biomedical context which provides unique physiologic information, as hemodynamic variations in the organ's microcirculation and alterations in oxygen supply, demand and consumption in the detrusor muscle can be monitored in real time [80,82,102]. Such information enhances investigation of patients with bladder dysfunction, offers new insights into causal pathology and thus can contribute to both diagnosis and selection from therapeutic options. As with most other NIRS applications the data obtained are not available by other means [40,90,102], and the physiologic effects inferred can contribute important insights when used alone or interpreted in parallel with other conventional measurements $[102,112,126]$.

In becoming an established non-invasive optical technique NIRS has continually benefited from advances in the hardware and software available, and the broad expertise that exists with the technology reflected in the substantial scientific literature available [27,37,43,53,104,133,142]. Continuous wave (CW) NIRS instruments have principally been used in urology [76,79]. Initially NIR light was lasergenerated; now miniaturized self-contained devices using light emitting diodes, spatial configuration of multiple emitters to the detector, and wireless capacity enhance research scope and clinical monitoring potential. In addition to monitoring changes in hemoglobin concentration from baseline, which allow changes in tissue hemodynamics and oxygenation to be inferred, spatially resolved (SR) instruments now provide an absolute measure of tissue oxygen saturation [83,117,127].

Bladder spectroscopy generates patterns and trends in chromophore change as the organ fills and empties that differ in health and disease $[38,77,79,80,84,102]$. Such data are physiologic because NIRS only 
detects change during events in the voiding cycle; pathologic changes mirror NIRS-derived effects of physiologic events such as hypoxia, ischemia and fatigue observed in other tissues, and animal data and independent research corroborate the principal findings $[72,81,82]$. In addition to adding new knowledge relevant to the underlying cause of voiding dysfunction, construct of algorithms utilizing chromophore parameters characteristic of specific pathology has resulted in NIRS monitoring having comparable discriminant ability to the current invasive diagnostic method, urodynamic pressure flow studies (UDS) $[40,85,121,129,144]$.

The goal of this review is to summarize the evolution of NIRS monitoring to study the urinary tract, testes and pelvic floor, and focus on biomedical applications of NIRS to study the human bladder. It describes the rationale for monitoring the bladder using NIRS, the basic science principles and specification of the instruments employed, how data are measured and interpreted, our principal research findings and parallel work by other investigators, why the data obtained reflect physiologic changes in the bladder, and future directions for bladder monitoring using NIRS.

\section{Innovation with NIRS in urology}

Prior literature provides reports from individual animal and human studies, reviews of applications of NIRS in the field of urology $[72,79,82,125]$, and descriptions of the development of dedicated instruments for bladder studies and recent introduction of wireless devices $[76,86,99]$. Urologic conditions studied include testicular hypo-perfusion, erectile dysfunction, renal hypoxia, skeletal muscle metabolism in patients with end stage renal disease, and the toxic effects of renal contrast media. Investigation of voiding dysfunction has included animal and human bladder studies and monitoring of the urinary sphincter and pelvic floor to establish the NIRS changes observed during normal voiding and the effects of a variety of pathologies that cause problem voiding in adults and children. Detection of ischemic change in the human testis as a diagnostic measure for testicular torsion and increased vascularity of the bladder wall secondary to inflammation/infection are the most recent innovations [20,113, $116]$.

\subsection{Initial applications}

Colier first used NIRS to study testicular hemodynamics in 10 boars by measuring active testicular blood volume using a CW instrument before and after spermatic cord occlusion [26]. NIRS combined with pulse oximetry was proposed as a way to quantify changes in testicular blood volume in real time as a measure of the viability of an intra-abdominal testis. Capraro used a CW NIRS derived measurement of testicular tissue oxygen saturation $\left(\mathrm{StO}_{2}\right)$ in sheep to investigate the effect of experimental testicular torsion and surgical relief of torsion [23]. The study showed sensitive detection of testicular hypoxia following torsion and also reperfusion of the hypoxic testis after torsion was reduced. The same parameter and comparable methodology were also used to study a rat model [6]. A dual wavelength CW NIRS device was used to compare the ratio of the average intensities of transmitted light at 660-940 nm to diagnose torsion induced in rats; this device employed LEDs as the light source [22]. And, absolute values of testicular $\mathrm{StO}_{2}$ were obtained using a frequency domain spectrometer with a specially designed optical probe in a similar study in rabbits [52].

Penile spectroscopy was performed on patients with erectile dysfunction and normal volunteers to study the physiology and vascular properties of the penis related to erection [21,95]. A CW device (wave- 
length $805 \mathrm{~nm}$ ) with a customized probe was used with simultaneous color Doppler ultrasound (CDU), strain gauge penile circumference monitoring and penile tonometry. Penile blood volume changes over time were documented following intra-cavernous stimulation. The conclusion was that NIRS can evaluate hemodynamic phenomena in the penis and discern erectile end-organ failure [21]. However, the availability and acceptance of current pharmacological treatments for erectile dysfunction that enhance the effects of nitric oxide effectively obviates the need for comprehensive investigation.

The effect of hypoxic episodes on cerebral and renal tissue oxygenation was studied in mechanically ventilated preterm neonates via emitter/receiver patches placed on the forehead and the skin over the right kidney. During episodes of decreased oxygen saturation $\left(\mathrm{SaO}_{2}<80 \%\right.$ for $\left.>4 \mathrm{~s}\right)$ fractional oxygen extraction in cerebral tissue was comparable to non-hypoxic controls, but increased oxygen extraction occurred in renal tissue. Advantages of NIRS identified were the ability to study infants at the bedside, the safety of light as an energy source, and the non-invasive nature of the technology [103].

Three studies addressed aspects of renal failure. Studies that combined MRI and NIRS were done on calf muscle in patients with chronic renal failure (CRF) on maintenance hemodialysis (HD) before and after administration of L-carnitine. $\mathrm{O}_{2} \mathrm{Hb}, \mathrm{HHb}$, and myoglobin $(\mathrm{Mb})$ concentration were monitored to derive measures of muscle bioenergetics before and after an exercise protocol (half-time for recovery of the NIRS signal as an indicator of tissue oxygenation and return of perfusion to resting level after exercise) [138]. Calf muscle was also studied with NIRS in HD patients and control subjects at rest, and during and after an exercise protocol, and muscle oxygenation and perfusion compared. It was concluded that muscle dysfunction in HD patients is related to a mitochondrial defect [62]. Forearm muscle studies in children with end stage renal disease showed their skeletal muscle oxidative metabolism during exercise was impaired, and improved remarkably after renal transplantation. Evaluating handgrip exercise allowed alterations in $\mathrm{Hb} / \mathrm{Mb}$ deoxygenation during arterial occlusion to be compared as an indicator of the rate of oxygen consumption in mitochondria, and recovery time as an indicator of muscle aerobic capacity [92].

Renal tolerance to contrast agents is a significant clinical issue and the effect of agents with differing osmolality were assessed in an animal model. A small NIRS probe was placed on the renal cortex of rats to measure alteration of $\mathrm{O}_{2} \mathrm{Hb}, \mathrm{tHb}$, and oxygen saturation after injection of iodinated contrast media, and after addition of a prostacyclin analogue (iloprost) [66]. Contrast agents had a negative effect on blood flow and oxygenation, and iloprost attenuated the decrease in oxygen saturation. Development of less toxic contrast media has long been a subject of research in uroradiology [61].

In fNIRS brain studies related to voiding a 52 channel NIRS machine has monitored changes in cortical $\mathrm{O}_{2} \mathrm{Hb}$ concentration as a measure of variations in frontal lobe blood flow over brain areas identified to be involved in the neural control of micturition [11] via fMRI, PET and SPECT [14,45,49]. Changes in $\mathrm{O}_{2} \mathrm{Hb}$ concentration before and after voiding, and during compression of the lower abdomen to simulate the urge to void were compared in control subjects [93]. Adult controls and subjects with detrusor over activity (DO) have also been compared using fNIRS. During natural filling to the point just after voiding began, a continuous increase in $\mathrm{O}_{2} \mathrm{Hb}$ concentration was evident in the frontal micturition areas in control subjects, followed by a continuous decrease after voiding. In contrast, in subjects with DO it was rare to see an increase in $\mathrm{O}_{2} \mathrm{Hb}$ before the first sensation to void occurred, and frontal cortical activation was weak [109]. Combined NIRS-UDS studies show brain activation in OAB patients before and after anticholinergic drug administration [107,108].

Recently Burgu demonstrated the feasibility of using trans-scrotal NIRS measurement of testicular $\mathrm{StO}_{2}$ in adults to identify torsion by comparing the vascular status of the right and left testes in suspected cases [20]. The affected testis showed significantly reduced oxygenation at presentation, and 
improvement following surgery to relieve torsion. Measurement of a difference in spermatic cord oxygen saturation has been used as a diagnostic measure in a child [113]. A self-contained CW SR-NIRS device was apposed to the skin over each spermatic cord in turn for one minute, followed by similar comparison between the right and left quadriceps as a control measure.

Torsion is an emergency due to the risk of testicular loss, hence, once suspected, emergent surgical exploration has been considered mandatory [60]. Various diagnostic modalities are used to avoid unnecessary operative intervention (radionuclide imaging, color Doppler ultrasonography, high-resolution ultrasonography), but each has drawbacks [113], hence, further exploration of the use of NIRS is logical because of ease of use and proven ability of the technology.

\subsection{Instruments: Continuous wave spectroscopy}

Laser powered continuous wave (CW) instruments were used for the first applications in urology [75, 125]. The development of the first NIRS instrument designed specifically for bladder studies has been described [86]; and the subsequent evolution of the latest self-contained wireless technology we utilize reviewed $[76,79]$.

The early instruments used incorporated lasers which provided high spectral resolution and high sensitivity, [42] but these instruments tended to be large. Also the fiber optic cables necessary to connect the instrument to the emitter/detector patch on the skin constrained the patient. (Figure 1 illustrates the laser powered instruments used to develop bladder spectroscopy.) Some instruments allowed a choice from multiple wavelengths, and some offered more than one data channel which allowed comparison of different sites [44]. For example, we used a two channel device (Oxymon, Artinis BV, The Netherlands) [136] to confirm that signals occurring during voiding were only detected over the bladder and not from elsewhere over the abdomen [125]. As multiple channel instruments evolved, the capacity was added
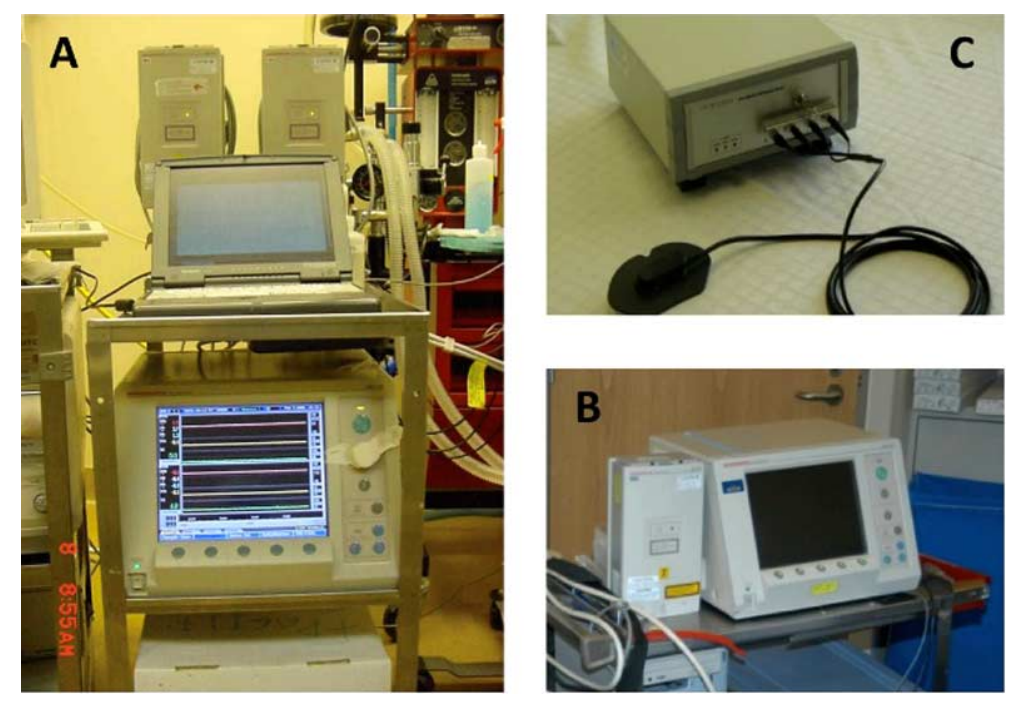

Fig. 1. Laser powered CW NIRS instruments used to develop spectroscopy of the bladder. (A) The first (NIRO 500, Hamamatsu Photonics KK, Hamamatsu city, Japan) required photomultiplier tubes for photon detection. (B) The second (NIRO 300, Hamamatsu Photonics KK, Hamamatsu city, Japan), a smaller multichannel device with photodiode detector. (C) The first instrument designed for bladder monitoring (URO-NIRS, Urodynamix Technologies, Vancouver, Canada) showing the fiberoptic cables and light occluding patient interface containing the emitter and detector. (Colors are visible in the online version of the article; http://dx.doi.org/10.3233/BSI-140091.) 


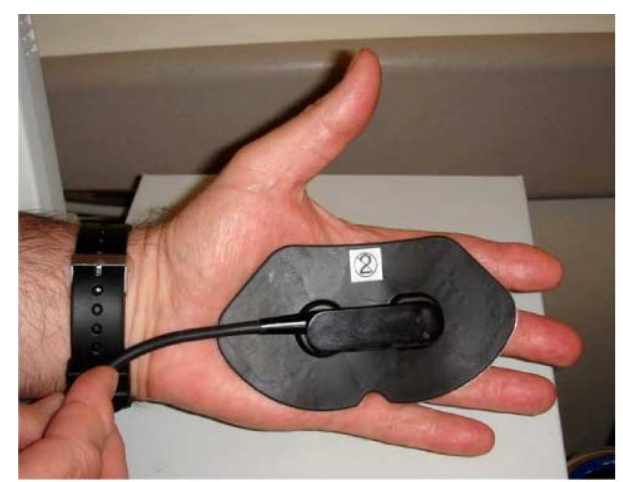

A

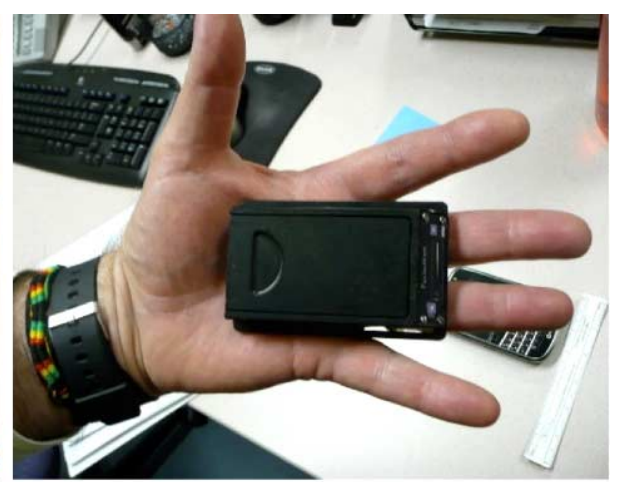

B

Fig. 2. (A) The optode patch (patient interface) of the original bladder NIRS instrument, and (B) a self-contained wireless device. The size comparison illustrates the progressive miniaturization possible with LED incorporation in NIRS devices. (Colors are visible in the online version of the article; http://dx.doi.org/10.3233/BSI-140091.)

for functional monitoring (fNIRS) using an array of multiple emitters and receivers [5,58]. This feature was used to display bladder data in one of our subjects in the form of real time video of hemodynamic change [78].

Light emitting diodes (LEDs) are now an attractive alternative to lasers. LEDs have minimal power consumption so can be powered by small batteries, and because the light source is non-coherent and non-collimated can provide high light intensity levels [17,79]. Very small self-contained wireless NIRS devices have been designed that make use of LEDs (Fig. 2), and such devices enable subjects to be monitored more simply than with laser powered instruments [76,77,80,83]. In urological applications the availability of such devices has made it possible for us to study small children, and to monitor ambulant patients over longer periods of time. Other investigators have used similar devices for biomedical applications that include sports science, athletic training evaluation, rehabilitation medicine, high altitude research, orthopedics, occupational health, evaluation of peripheral vascular disease, and studies of muscle function in subjects undertaking a range of active physical pursuits [76].

In the design of devices with LEDs elements of compromise have been required, particular for the detectors, batteries, and telemetric communication components in wireless systems. For example 'Bluetooth' capacity, while ideal for linking to remote data storage or mobile phones, does use more power than other wireless communication systems, which results in the need to incorporate a battery with higher capacity.

An important design element has been the ability to configure LED wireless devices with the optical geometry required for spatially resolved spectroscopy (SRS), as this enables an absolute measure of tissue oxygen saturation to be obtained $[83,91,127]$. The terms used for the oxygen saturation measurement obtained differ depending on the manufacturer of the device, and investigators and clinicians have learned that saturation values are not directly comparable between devices due to key differences, including variations in the software algorithms employed [72]. But measurements can be made in real time and are of recognized value as a means of detecting trends in tissue oxygen saturation.

CW SRS NIR instruments of small size and with telemetric capacity represent an important advance in the application of NIRS in both a research and clinical context. Such devices are now used in our laboratory and for clinical studies, as they monitor changes in bladder hemodynamics, oxygen supply and demand, and provide measurement of tissue oxygen saturation in the detrusor muscle $[76,79,83,112$, 


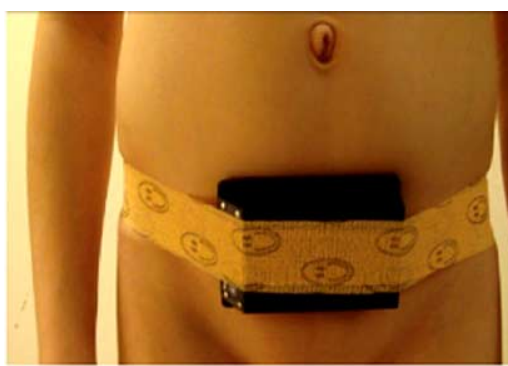

A

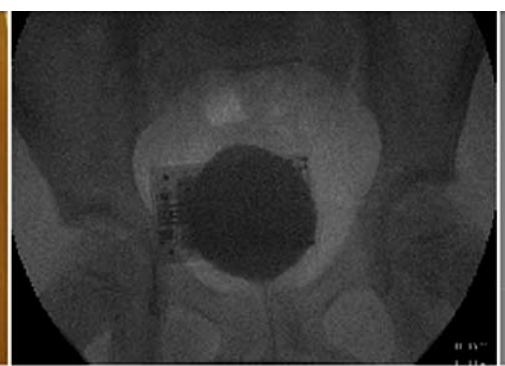

B

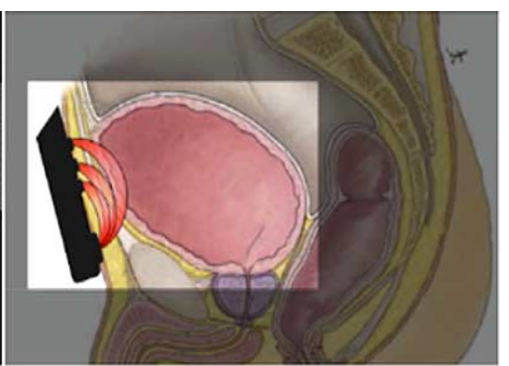

C

Fig. 3. (A) A wireless device (PortaMon) positioned for bladder monitoring on a child. (B) Radiographic confirmation of the position of the device over the bladder. (C) A diagram illustrating the 'banana' shaped photon path. (Colors are visible in the online version of the article; http://dx.doi.org/10.3233/BSI-140091.)

115,116]. The commercially available wireless device most widely used by our group for bladder NIRS is the 'Portamon' (Artinis Medical Systems BV, The Netherlands).

We have described the specifications and advantages of this device in reports of its use $[76,79,117]$. Key elements are that it is self-contained and of small size $(83 \times 52 \times 20 \mathrm{~mm}$, weight $84 \mathrm{~g})$ and incorporates 'Bluetooth' technology with broadcast range of 100 meters. Three pairs of LEDs (760 and $850 \mathrm{~nm}$ ) are configured for SRS with the single detector to provide 3 source-detector separation distances (30, 35 and $40 \mathrm{~mm}$ ). The detector is a silicon photodiode with a filter to provide ambient light protection. Data is collected at $10 \mathrm{~Hz}$. The rechargeable battery (Lithium Polymer) has a six hour capacity and the internal memory 2 megabytes for storage. Proprietary software ('Oxysoft', Artinis Medical Systems BV, The Netherlands) [136] allows data interpretation at each source-detector distance, derives changes in the concentration of the chromophores $\mathrm{O}_{2} \mathrm{Hb}, \mathrm{HHb}$ and $\mathrm{tHb}$ from the raw optical data, and an absolute measure of tissue oxygenation (expressed as tissue saturation index or TSI \%). The device is taped to the skin over the bladder (Fig. 3).

\subsection{Instruments: Additional spectroscopic techniques}

Other spectroscopic modalities used in urology have included fluorescence (reflection) spectroscopy (FS) which measures the amount of light reflected from a substance to detect differences in the fluorescent properties of pathologic tissues [15]. FS relies on differences in fluorescent emission of photons by different molecules when they are exposed to a monochromatic light source such as a laser. FS has been used to explore differentiation of normal from neoplastic epithelium, including differentiating normal bladder urothelium from transitional cell carcinoma. Although several reports have confirmed the high sensitivity of this technique the specificity is low.

Raman (scattering) spectroscopy (RS) measures the wavelengths that a substance or tissue reflects on excitation by laser light and generates unique Raman spectra in vitro that can be used to determine the composition of the tested sample $[9,68]$. RS provides detailed cellular analysis and an objective method for diagnosis of pathology such as cancer and infection, and in urology shows promise for in vitro diagnosis and grading of bladder and prostate cancers. RS can also be used to identify the composition of urinary stones and their effect on renal medullary collecting ducts through analysis of biopsies from renal papillae. New developments in RS will likely provide in vivo urological applications for guiding endoscopic procedures and assessing tumour resection margins. 
Time resolved (TR) spectroscopy measures the time of flight in addition to light intensity and is used experimentally to determine the optical properties of tissues, three-dimensional imaging and tomography [31]. TR is being explored experimentally to examine the optical properties of prostatic tissue in vivo in the context of developing photodynamic therapy as a modality for the treatment of prostate cancer [128].

Differential path length spectroscopy (DPS) has been used to measure microvascular oxygenation in the human bladder [3]. The technique involved is invasive, but measurements are possible on both the inside and outside surfaces of the bladder wall. In future, spectroscopic technologies such as DPS could be used in conjunction with transcutaneous CW NIRS to learn more about the microcirculation of the bladder; especially to determine whether disease affects both the muscle and the urothelial vasculature.

\subsection{Computer aided design, data analysis and diagnosis}

In urology, as with applications of NIRS elsewhere, the hardware and software available are continuously evolving.

Computer assisted design (CAD) is employed in the development of an increasing number of biomedical devices [88], and CAD and related programs are now being explored in the evolution of NIRS devices for urologic applications. The CAD process controls the development of a technical system to achieve an optimum balance of all the system's elements. Computer systems engineering (CSE) translates the design goals for a device into clearly defined system parameters, allocates these parameters to the various disciplines, and integrates the processes needed to realize the system. The resulting model can also have data from subsequent trials of the system fed back into it for further refinement.

Diagnostic algorithms using NIRS-derived criteria are now available in addition to the conventional algorithms required to process raw optical data, generate changes in chromophore concentration, and calculate SRS-derived measurement of oxygen saturation. In bladder voiding studies the opportunity was presented to develop such algorithms when characteristic patterns of change in NIRS parameters were identified to occur in symptomatic patients with bladder outlet obstruction (BOO) $[84,85]$. Ultimately, a diagnostic algorithm which utilized NIRS data alone was generated using classification and regression tree analysis (CART) of data from the entire voiding cycle; this was equally discriminant for the presence or absence of BOO as invasive studies [51,121]. Classification and regression trees are machine-learning methods that construct prediction models by partitioning data and fitting a simple prediction model within each partition [19]. The resulting model can be represented as a decision tree. Classification trees are designed for dependent variables that take a finite number of unordered values. In contrast, regression trees are for dependent variables that take continuous or ordered discrete variables. The binary prediction trees that result can be further improved by inclusion of multiway splits [71]. In our experience CART is particularly suited to modeling the non-linear data NIRS generates.

\section{NIRS principles employed in urological studies}

There are comprehensive reviews of the applicable science related to NIRS, the principles underlying monitoring and measurement of quantifiable parameters, and the limitations of the technology for clinical monitoring [13,16,27,41-44,53,142]. The unique properties of NIR light have been central to urological studies. In particular, its penetration through skin and scattering in tissue, with recognition that depth of penetration is a factor of the inter-optode distance (IOD), and that there are wavelength depen- 
dent differences in absorption by hemoglobin when the molecule is oxygenated $\left(\mathrm{O}_{2} \mathrm{Hb}\right)$ or deoxygenated (HHb) $[44,54,135]$. The instruments used have incorporated NIR wavelengths matched appropriately to the peak absorption of the chromophore(s) of interest, which in the case of changes in hemodynamics and oxygenation in the bladder are $\mathrm{O}_{2} \mathrm{Hb}$ and $\mathrm{HHb}$. Hence most instruments used for bladder studies have wavelengths between $729-875 \mathrm{~nm}$, although some utilized included $920 \mathrm{~nm}$. There is now interest in higher wavelengths, as $975 \mathrm{~nm}$ corresponds to the peak absorption for water [29]. While this wavelength is usually avoided during bladder NIRS so that physiologic changes in the anterior bladder wall can be monitored without confounding absorption by the urine in the bladder, this wavelength has specific relevance to us as a means of monitoring change in bladder size in real time. This can be achieved by detecting altered attenuation at $975 \mathrm{~nm}$ as the bladder enters the field of view of a NIRS device placed on the abdomen so that it can monitor when the volume of urine in the bladder reaches capacity [99]. The software algorithms based on modification of the Lambert-Beer law continue to be utilized with custom refinements for some display requirements, including for fNIRS [32,42]. These accommodate for limitations posed by the nature of human tissue, including where the path length of NIR light and number of photons lost due to scattering are unknown.

In urological studies, real time graphics showing increases and decreases in $\mathrm{O}_{2} \mathrm{Hb}$ and $\mathrm{HHb}$ and their relationship to one another have provided us with valuable insight into the provision and consumption of oxygen, even though conventional $\mathrm{CW}$ instruments can only measure changes in concentration relative to the initial baseline because the full extent of the field through which NIR light scatters is always unknown in vivo $[16,44,142]$. These parameters are a measure of the balance of oxygen supply to metabolic demand, while total hemoglobin concentration $(\mathrm{tHb})$ reflects changes in the volume of blood in the tissue field interrogated. Now, in addition, spatially resolved spectroscopy provides an absolute measure of tissue oxygen saturation. SRS incorporates two or more NIR light emitters positioned at different distances from the photodiode detector which allows measurement of light intensity as a function of distance; with appropriate algorithms the ratio of oxygenated to total tissue hemoglobin is then calculated from which an absolute measurement of tissue oxygen saturation is made [83,91,105,127]. These values predominantly reflect venous oxygen saturation as only the minority of the blood in tissue is in capillaries and arterioles; and in SRS the assumption is made that the tissue interrogated is homogeneous.

Monitoring the redox status of cytochrome-C-oxidase (CCO) would theoretically provide additional information relating to electron transport and oxidative phosphorylation at a cellular level in the bladder, as the organ's mitochondria contain significant quantities of CCO. However, we have only proved the feasibility of monitoring bladder cytochrome redox status in one bladder study to date (Fig. 4), and one involving the muscles of the pelvic floor (Fig. 11) [122,124]. CCO drives $>95 \%$ of $_{2}$ consumption and adenosine triphosphate (ATP) synthesis, and its redox status has principally been monitored in the brain $[28,74,119]$. The incentive to monitor $\mathrm{CCO}$ redox status is that while the $\mathrm{O}_{2} \mathrm{Hb} / \mathrm{HHb}$ relationship indicates whether oxygen has been delivered and/or depleted, $\mathrm{CCO}$ changes reflect more intricate physiologic interplay such as the presence or absence of oxygen uptake, cellular transition from oxidative to substrate dependent phosphorylation, and whether or not glucose is available or ATP synthesis impaired [75]. However, monitoring is a technical challenge because the contribution of CCO to the overall absorption of NIR light is only about one tenth that of hemoglobin [29].

Validated methodologies exist for a range of NIRS-derived quantitative measurement parameters [42, 142]. While principally from the large body of research in animals and humans that relate to the study of brain and voluntary muscle, some are already applicable to urology $[72,124]$. 


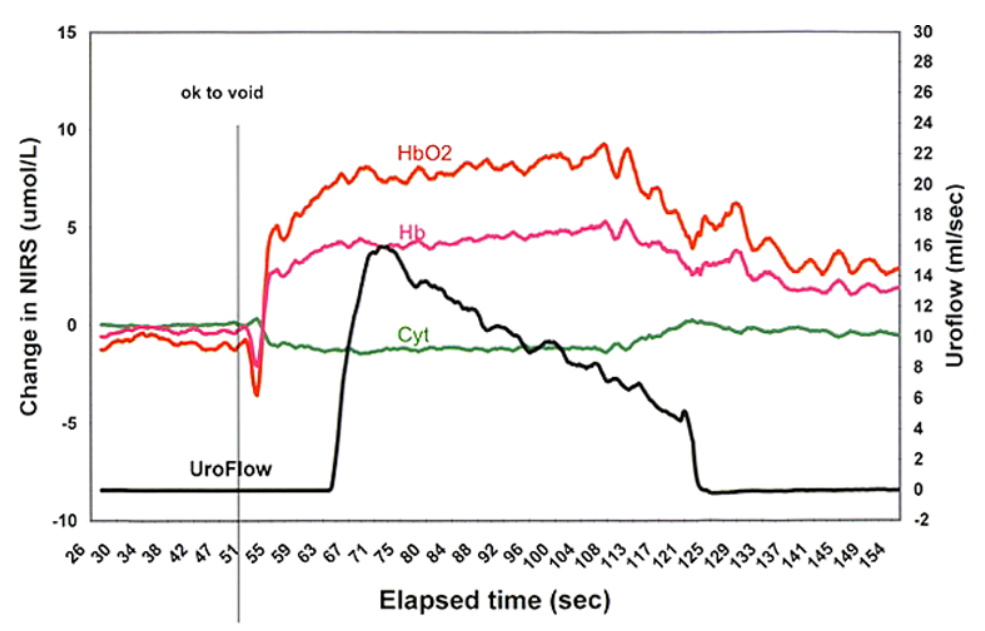

Fig. 4. A graph of chromophore concentration changes occurring during normal voiding in an asymptomatic adult - oxygenated and deoxygenated hemoglobin $\left(\mathrm{O}_{2} \mathrm{Hb}, \mathrm{HHb}\right)$ and cytochrome (Cyt). This shows a shift in the equilibrium of cytochrome redox status occurring in relationship to bladder contraction and urine flow (uroflow) following permission to void (ok to void). (Colors are visible in the online version of the article; http://dx.doi.org/10.3233/BSI-140091.)

\section{The relevance of NIRS for bladder monitoring}

Bladder disease and related problems with urination constitute a major health problem which negatively impacts quality of life $[67,120]$. Over one million new patients present for evaluation annually in the USA alone, no age group is immune, and worldwide numbers are increasing amongst aging populations [33]. Problematically the current 'gold standard' method for evaluation of bladder dysfunction, urodynamic pressure flow studies (UDS), is recognized to have limitations. This test only provides values for the pressure within the bladder and rate and volume of urine flow, and generates little direct information about the causal pathology underlying the voiding dysfunction. UDS testing involves a number of elements and is an invasive procedure that requires placement of catheters into the bladder and rectum for measurement $[48,72,101]$. Having a catheter in the urethra compromises the physics of flow; there are significant health risks associated with catheterization; and the invasive nature of this investigation makes many patients decline to have it done. Hence the value of a non-invasive methodology that provides physiologic information and the potential for diagnostic algorithms.

The bladder is an ideal organ for spectroscopic monitoring because of the nature of its blood supply, consistent anatomic location in the lower abdomen and proximity to the skin [73]. The site of the emitter/detector on the abdomen used for interrogation of the bladder is also optimal, as many recognized confounders for NIRS are absent. There are not multiple layers of tissue/bone/fluid with different attenuating properties between the emitter/detector and the anterior bladder wall. And, even subcutaneous fat is minimal (in the order of $8 \mathrm{~mm}$ ) [126], which is an advantage for bladder NIRS as it is known from studies in other tissues that the thickness of the fat layer can compromise transmission of NIR photons to and from deeper tissue of interest [16,94]. However, muscle studies indicate that NIR photons tend to pass through adipose tissue as it has a low scattering coefficient and significantly lower absorption coefficient providing an adequate IOD is used [16,131]. The anatomy, vasculature, contractile properties and normal function of the bladder, and mechanisms underlying impairment of urine storage and drainage relevant to NIRS studies have been reviewed [73]. 


\subsection{Anatomy}

The anatomical consistency of the bladder's position is dictated by the attachment of the organ's base within the pelvis [25]. Stothers et al. [126] reported ultrasound data showing that the anterior wall retains its position in relation to the abdominal wall as the organ fills and empties, with a mean depth below the abdominal skin of $2.7 \mathrm{~cm}$ (range 1.24-3.95).

\subsection{Vasculature}

The bladder is richly vascularised [25,73,110]. Its microcirculation has unique architecture and autoregulatory mechanisms that make consistent perfusion possible by accommodating for the bladder wall stretching and thinning during filling and contracting and thickening during voiding, mechanical changes that would compromise blood flow in the absence of an adaptive microcirculation $[18,56,63,97$, 137]. Importantly, various pathologies associated with bladder dysfunction and aging are known to compromise this adaptive ability $[4,7,10,65,70,87,141]$; a possible mechanism is endothelial dysfunction and impairment of nitric oxide synthesis $[30,137]$, and NIRS data show important physiologic differences occur in detrusor blood volume and oxygenation in health and disease.

\subsection{Contractile properties}

During urination the detrusor muscle contracts over a large length interval but at other times remains relaxed which allows the bladder to fill [4,50]. The detrusor's smooth muscle fibres are in spiral, longitudinal and circular bundles in the bladder wall in close proximity to the arterioles and venules of the microcirculation. The urinary sphincter also has a rich neurovascular network and its function is integral to normal bladder filling, retention of urine, and voiding especially in females [47]. Muscles enveloping the urethra also contract to control urine flow.

\subsection{Normal bladder function}

In health, the bladder microcirculation effectively coils and uncoils the vessels in the microcirculation during contraction and expansion respectively to maintain tissue oxygenation as metabolic demands change [18]. This compliments neuronal, endothelial and erythrocyte mediated signalling pathways that integrate smooth muscle and endothelial cell function, determine convection and diffusion of oxygen, and dictate where blood flow is distributed via the microcirculation [36,104,111]. During normal filling and expansion of the bladder, the straightening and stretching of vessels that occurs results in decreased resistance and increased blood flow despite an increase in bladder wall tension. Kershen et al. [63] have shown that during the filling phase bladder blood flow increases approximately 1.8 times over that of the empty bladder. Also, that immediately after the bladder empties, a rebound increase in flow occurs, raising blood flow to about 1.6 times that of the empty bladder.

\subsection{Impaired storage and voiding}

Voiding dysfunction is a significant health burden [33,120]. Affected patients experience a range of lower urinary tract symptoms as a result of a number of systemic and local pathologies. Importantly, in the context of bladder spectroscopy, one causal mechanism shared by several pathologies is that perfusion in the main bladder muscle, the detrusor, becomes abnormal. From our studies we now believe that the common pathway for this abnormality is an adverse effect on the hemodynamic control of the detrusor microvasculature, which either compromises the provision of an increase in oxygenated blood 
required for normal detrusor contraction, and/or results in an imbalance in oxygen supply and demand as voiding occurs [82].

The impact of disease on the hemodynamics and oxygen supply of the bladder makes NIRS relevant as a means of assessing bladder function. A variety of patterns of chromophore change have been observed in patients with dysfunctional voiding, and significantly these match patterns seen in other tissues where hemodynamic abnormalities are also present, and/or there is disordered oxygen supply and demand. At their most extreme, hemodynamic changes we have observed using NIRS even imply that in some severely symptomatic patients their bladders become ischemic during voiding, ultimately followed by reperfusion [77,80]. This correlates with changes that can occur with aging [87] and experimental studies where marked changes in detrusor compliance and contractility are associated with chronic bladder ischemia $[7,8]$. Ischemia may also have a role in bladder neuropathy as noxious oxidative products have been detected where overactivity occurs under ischemic conditions.

\section{How the bladder is monitored using NIRS}

NIRS is an ideal monitoring technology for the bladder, because real time changes in hemodynamics and oxygenation can be detected as the bladder fills and empties [38,40,77,78,80,82,83,85,102,140, 144]. The site used for optimal interrogation of the bladder is on the skin of the lower abdomen. The emitter detector array configured in reflectance mode is placed across the midline $2 \mathrm{~cm}$ superior to the pubis (see Fig. 3) [80,86]. Transcutaneous monitoring can be conducted during bladder filling and during spontaneous voiding as the bladder is emptied. Voiding involves contraction of the detrusor muscle in the bladder wall, when, as would be expected from NIRS studies of voluntary muscle [57,130,142], changes in chromophore concentration are readily observed. Bladder NIRS can also be conducted simultaneously with invasive urodynamic measurements of pressure and flow, and use of other technologies such as ultrasound and fluoroscopy.

\section{CW NIRS monitoring of the bladder}

\subsection{Historical perspective}

The place of happenstance in research is under-stated. Understandably, 'cutting edge' publications report advances, and 'state of the art' reviews emphasize the progression and fundamental logic of what occurred. But bladder NIRS would not have evolved without a chance finding during animal studies exploring the effects of cardiac bypass pump flow on cerebral hemodynamics, where a second NIRS channel monitoring the abdomen as a control measure intermittently showed significant chromophore change. We eventually determined these changes were temporally related to the bladder emptying and, although as Pasteur said (generously), "Chance only favors those minds that have been prepared", the significance of this could have escaped us had a urologist not been in the lab at the time. Formal animal studies to confirm the finding followed, and it became clear with these, and subsequent human trials, that the filling and emptying phases of voiding were physiologically rich events where NIRS could detect a range of hitherto unavailable data.

The evolution of bladder spectroscopy that followed was an iterative process influenced by the evolution of NIRS devices and software, experience gained from research, and growing awareness of how NIRS could contribute to clinical assessment and basic science research in urology. 
A series of reviews [72,79,80,82] and research papers [39,40,77,83,85,90,112,121,129,140,144,145] describe the major findings related to bladder NIRS. We first reported the feasibility of using NIRS in the evaluation of bladder function by describing trans-abdominal NIRS of the bladder in an adult patient during voiding where a temporal change between $\mathrm{O}_{2} \mathrm{Hb}$ and $\mathrm{tHb}$ concentration was demonstrated during voiding [75]. In subsequent studies we explored the potential of non-invasive transcutaneous NIRS to study bladder filling and emptying in normal subjects [123] and distinguish between specific urinary pathologies using the patterns of chromophore change generated via NIRS during simultaneous urodynamic studies. Most recently absolute changes in detrusor oxygen saturation have also been compared in health and disease measured via a tissue saturation index (TSI \%) using a wireless SR NIRS device $[77,79,83,112,114-116]$.

\subsection{Study technique}

The methodology has been described for simultaneously monitoring the bladder with NIRS during UDS pressure flow studies, and to follow changes in detrusor hemodynamics and oxygenation during natural filling and voiding $[79,80]$.

The first devices used were laser powered instruments where the emitter and detector were housed in an adhesive light occluding patch placed on the abdominal skin over the bladder, and connected to the NIRS device by a fiber optic cable [86]. In early trials it was established that $4 \mathrm{~cm}$ IOD enabled the detrusor muscle in the anterior wall of the bladder to be interrogated in all but the most obese subjects. Later ultrasound studies confirmed the depth of the anterior wall below the skin and the relatively constant position of the bladder in the pelvis [126]. Now with current self-contained wireless devices the same site on the abdomen is used ( $2 \mathrm{~cm}$ above the symphysis pubis) but the whole device is located in this position, with the light emitters and photodiode detector placed across the midline [112].

A unique feature of NIRS is the ability to collect data during natural filling and voiding, i.e. without the presence of catheters in the urethra/bladder and rectum. The study process has been described $[79,80]$. Key elements incorporated include: baseline data collection for 30 seconds, followed by an instruction to the patient to empty the bladder ('permission to void'), spontaneous voiding into a uroflow meter (to record urine volume), and post-voiding data collection for one minute. The time points for permission to void and the start and end of urine flow are recorded, and the volume of urine voided. Ultrasound measurement of urine volume in the bladder pre and post voiding is an optional additional measure.

Also, NIRS is able to detect physiologic changes that occur in the bladder prior to the point that urine flow begins, i.e. between when a subject decides to void (or the command 'permission to void' is given during studies) and when urine flow starts. Importantly this phase of voiding is a time in the voiding cycle when invasive UDS tracings are 'silent' as no flow is occurring, but where we have learned through NIRS that significant physiologic events occur. These relate to changes in the microcirculation of the detrusor muscle, presumably triggered by signals to initiate voiding that originate in the brain's micturition centres, that increase blood flow and oxygenation in preparation for bladder contraction to take place $[77,79,82]$.

\subsection{Data display}

NIRS data can now be displayed in real time and in a number of ways, a measure of the progress from the time consuming spread sheet process used originally. Device software provides for graphic display of changes in concentration of individual chromophores and their sum total hemoglobin (tHb) against time. 


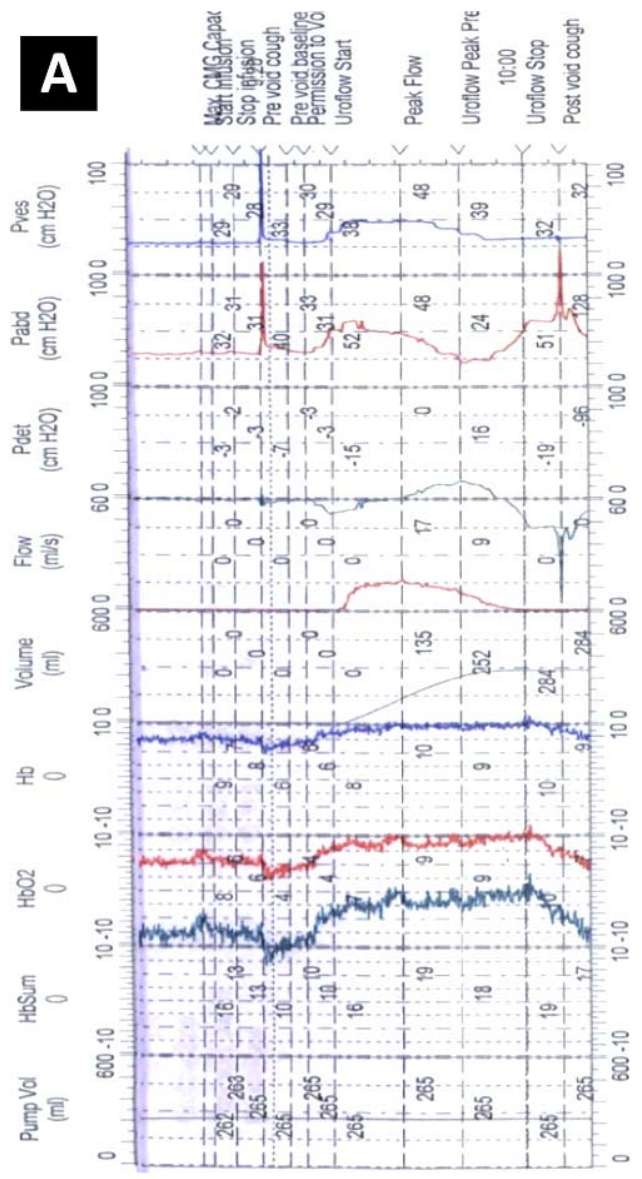

\section{B}

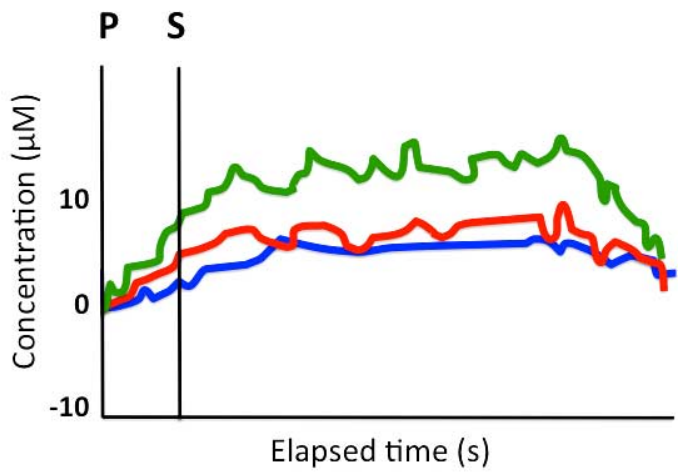

Fig. 5. A composite graph of a combined printout of simultaneously collected UDS parameters and NIRS data during voiding (A). With an inset graphic (B) of the NIRS-derived changes in $\mathrm{O}_{2} \mathrm{Hb}$, $\mathrm{HHb}$ and tHb concentration extracted and biased to show the patterns of chromophore change from permission to void (P), through the start of uroflow (S) and during voiding. (Colors are visible in the online version of the article; http://dx.doi.org/10.3233/BSI-140091.)

Although convention varies, the colors for display are usually red for $\mathrm{O}_{2} \mathrm{Hb}$, blue for $\mathrm{HHb}$, and green for tHb. NIRS parameters can be displayed simultaneously with data streams from other devices e.g. from UDS during voiding studies where it is important for data graphics from NIRS and UDS pressure/flow data to be accurately related to each other in terms of timing, so that they can be linked to symptoms and events occurring during the voiding cycle. However, the resulting composite graphs are complex (see Fig. 5).

For this reason, we have chosen to plot $\mathrm{O}_{2} \mathrm{Hb}, \mathrm{HHb}, \mathrm{tHb}$ separately, and bias the data at a specific point (e.g. permission to void), in order to make NIRS data trends and relationships easier to interpret. Examples of data displayed in this way are shown in Figs 5-7. Tracings can also be filtered to smooth the graphics generated. Such techniques makes it easier to see the interrelationship and potential relevance of NIRS data changes that relate to events in the voiding cycle, and to make comparisons between studies. Another early lesson learned was that care must be taken on composite graphs to have a sufficiently large scale for changes in chromophore concentration to be visible. 
A
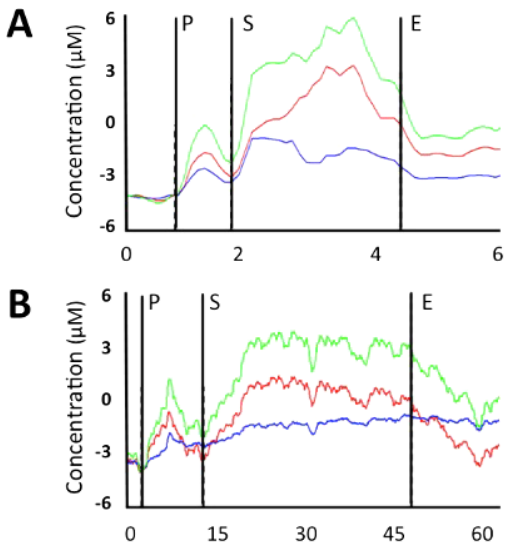

P: Permission to void

S: Uroflow start

C

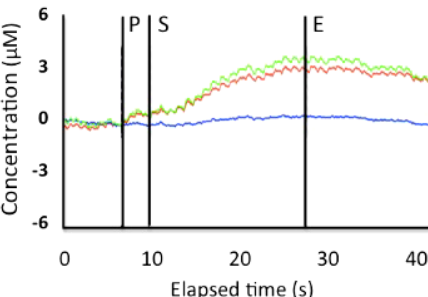

E: Uroflow end

$\mathrm{HHb}$

$\mathrm{tHb}$

$\mathrm{O}_{2} \mathrm{Hb}$

Fig. 6. Three examples illustrating the patterns of chromophore change seen in asymptomatic subjects during voiding ((A) a 7 year old girl, (B) a 31 year old male, and (C) a 42 year old female). An increase in $\mathrm{O}_{2} \mathrm{Hb} / \mathrm{tHB}$ occurs following 'permission to void' $(\mathrm{P})$, and a positive trend in $\mathrm{O}_{2} \mathrm{Hb}$ and tHb follows the start of uroflow $(\mathrm{S})$ that is sustained during bladder contraction to, or beyond, the point of maximum urine flow. (Colors are visible in the online version of the article; http:// dx.doi.org/10.3233/BSI-140091.)

\section{Principal research findings}

\subsection{Patient population studied}

Our study population has been broad. We have studied asymptomatic subjects, and patients of both genders with a broad range of symptoms, whose ages ranged from 5-78 years. Almost all patients with voiding dysfunction can be studied using NIRS, and the non-invasive optical nature of the technology leads to ready acceptance by patients. High body mass Index (BMI) is a potential limiting factor as NIR light penetration is influenced by body fat $[94,131]$. However, we have had very few situations where data could not be collected in properly conducted studies with fully functional equipment, as the monitoring site $2 \mathrm{~cm}$ above the pubis is below the abdominal fat pad in most obese subjects, and in muscle studies incorporating an adequate optode separation NIR photons tend to pass through adipose tissue an allow interrogation of deeper structures [16]. But, the thickness of the fat layer can ultimately compromise NIRS monitoring of deeper structures [16,131], and a BMI in excess of $30 \mathrm{~kg} / \mathrm{m}^{2}$ has been suggested by other investigators as potentially precluding bladder monitoring. Hematuria is also a contraindication for bladder NIRS as the absorption of NIR light by hemoglobin in the urine is a potential confounder [125].

\subsection{Asymptomatic (normal) subjects}

It has become clear that NIRS bladder data from asymptomatic children and adults are novel as they provide previously unavailable information on bladder physiology and function. During voiding, imme- 

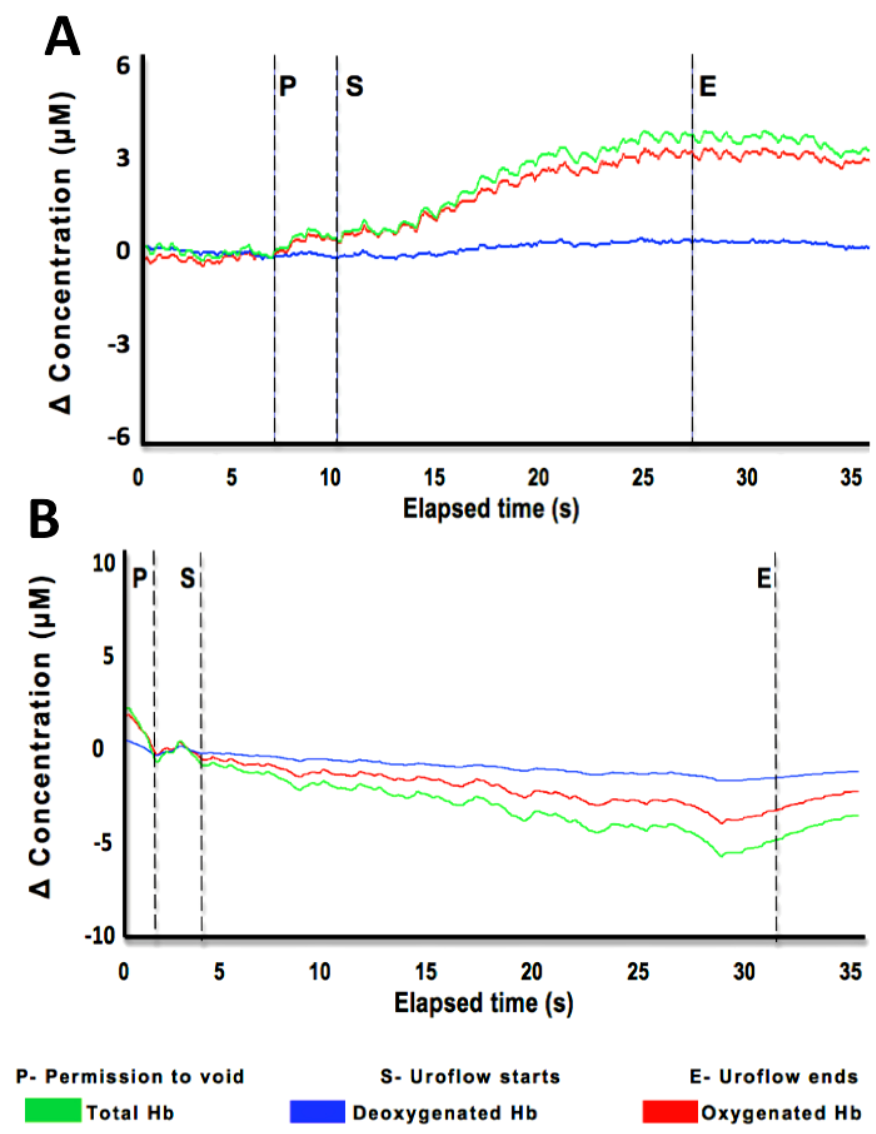

Fig. 7. Examples of the contrasting positive pattern of change in voiding chromophore data in an asymptomatic adult male subject (A), and the negative trend typical in bladder outlet obstruction (BOO) (B). (Colors are visible in the online version of the article; http://dx.doi.org/10.3233/BSI-140091.)

diately following the decision to void, a positive trend in $\mathrm{tHb}$ is seen that predominantly reflects a rise in $\mathrm{O}_{2} \mathrm{Hb}$. A further increase in blood volume/oxygenated hemoglobin supply then usually occurs as the flow of urine begins, while the $\mathrm{HHb}$ concentration remains essentially unaltered between the start and end of urine flow. Interpreting these data based on NIRS studies on voluntary muscle where responses within the microcirculation provide an increase in oxygenated blood prior to and during the work of contraction, we proposed that this sequence of changes indicated that:

- The bladder detrusor muscle is also 'primed' prior to contraction by provision of additional oxygenated blood via the microcirculation;

- the microcirculation continues to supply more $\mathrm{O}_{2} \mathrm{Hb}$ and greater blood volume during the work of voiding, and

- oxygen supply is greater than oxygen demand throughout the voiding cycle, as observed in voluntary muscle $[12,57,133]$.

Examples of this pattern of change are shown in Fig. 6 and demonstrate the reproducibility of these NIRS data between asymptomatic subjects, in spite of the variations inherent to individual voiding (duration, urine volume, pressure generated and flow rate). In contrast, studies we and others have reported 
identify that different patterns of change in $\mathrm{O}_{2} \mathrm{Hb}$ and $\mathrm{HHb}$ concentration are seen in symptomatic subjects with various diseases which imply that abnormalities in detrusor muscle hemodynamics or oxygenation are associated with symptoms of voiding dysfunction.

\subsection{Bladder outlet obstruction}

In 2007 Macnab and Stothers [84] reported an association in males with lower urinary tract symptoms (LUTS) between trends in NIRS parameters during dysfunctional voiding and a UDS diagnosis of bladder outlet obstruction (BOO).

Later results showed that the predominant NIRS pattern in BOO was a negative trend in $\mathrm{tHb}$ often associated with a fall in $\mathrm{O}_{2} \mathrm{Hb}$ during voiding (Fig. 7) [85]. This negative trend implies a reduced or absent hemodynamic response and/or a reduction in the availability of oxygenated blood during detrusor contraction $[79,80]$. In contrast, most of those not classified as having BOO using the UDS diagnostic nomogram (Abrahams Griffiths) [2] had NIRS patterns of change similar to those seen in asymptomatic subjects, showing some degree of positive trend in $\mathrm{tHb}$ and/or $\mathrm{O}_{2} \mathrm{Hb}$.

NIRS data indicative of similar hemodynamic abnormalities as we observed in BOO are also seen in tissues other than the bladder. In muscle, for example, such changes are reported where there is an unmet increase in metabolic demand, and/or impairment of the normal response of the microcirculation $[12,16,34,41,100,130,133,142,143]$. Under these circumstances, the functional capacity of the muscle or organ is adversely affected and, during work involving contraction, symptoms of dysfunction result. Based on such comparison we proposed the probable relationship between pathologies involving the bladder, symptoms indicative of detrusor muscle dysfunction, and NIRS changes reflecting impaired hemodynamics and/or oxygenation.

Next we constructed algorithms to compare the discriminant ability of NIRS-derived voiding data against the results of invasive UDS testing, recognizing that comparison with current 'gold standard' methodology is an obligatory step in developing any potentially disruptive technology. Although not the original intention, it became clear that NIRS-derived algorithms could be used for diagnosis when we found comparable discriminant ability between them and the nomogram used currently in UDS pressure flow studies to classify symptomatic males as having either an obstructed or unobstructed bladder [2]. Our first algorithm combined NIRS trend data $\left(\mathrm{tHb} / \mathrm{O}_{2} \mathrm{Hb}\right)$ with two non-invasive UDS parameters (residual volume of urine post voiding (PVR) and maximum urine flow rate (Qmax)). The sensitivity and specificity obtained were $87.7 \%$ and $88.9 \%$ respectively [85]. A second algorithm was created that used classification and regression tree (CART) analysis of chromophore data alone from the whole voiding cycle which had $88 \%$ specificity and $94 \%$ precision $[51,121]$. CART is used for mathematical modelling of non-linear components $[19,71]$ and hence is well-suited to NIRS data. Importantly, independent investigators have obtained comparably discriminant results using NIRS/UDS data algorithms; to date published reports include use of the original algorithm $[129,145]$ and two others that were similar, but independently derived [40,144].

The diagnostic ability of these algorithms strengthened our hypothesis that a disorder of detrusor hemodynamics is the principal physiologic anomaly underlying the symptoms associated with bladder outlet obstruction. Diagnostic discriminant ability with the degree of reproducibility demonstrated between 5 studies would also be unlikely if change in bladder size or other artefact were to influence NIRS-derived data. However, while an abnormal hemodynamic response during voiding is the predominant finding (Fig. 8 graph A), abnormalities in oxygen supply and demand can also occur as the detrusor contracts (Fig. 8 graph B) [80]. Chromophore changes indicative of detrusor hypoxia have been seen 
A

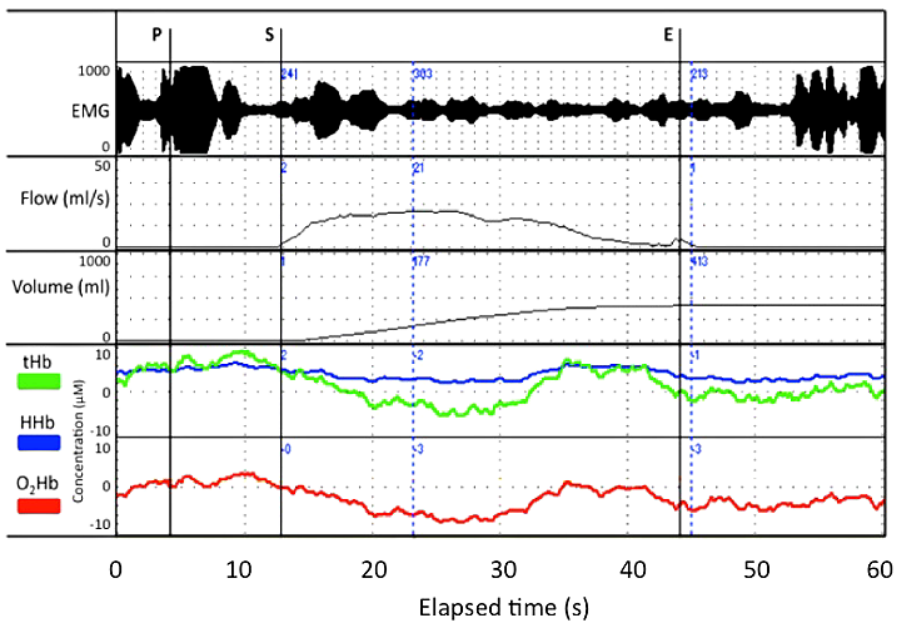

B

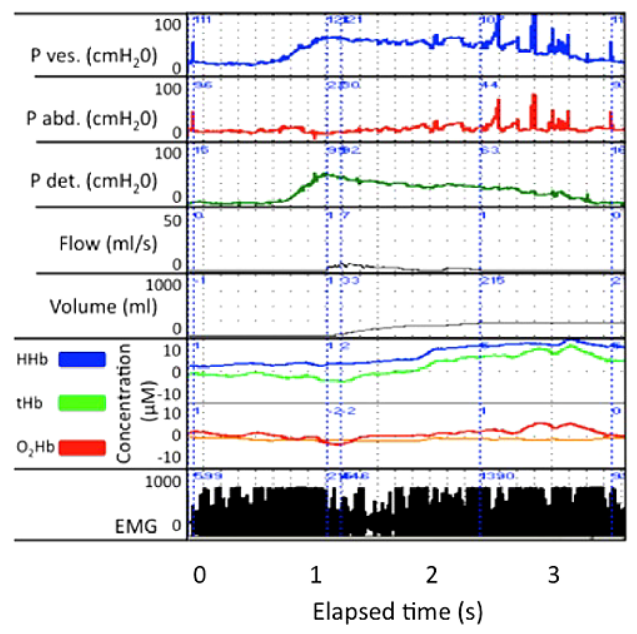

Fig. 8. A composite graph of NIRS and UDS data from males with BOO. In (A), a decrease in $\mathrm{O}_{2} \mathrm{Hb}$ and tHb begins prior to uroflow start and this negative trend continues up to the point of peak urine flow. This implies a decreased hemodynamic response and impaired provision of oxygenated blood to the detrusor during the first two thirds of the voiding cycle. In (B), $\mathrm{HHb}$ and $\mathrm{tHB}$ rise progressively during efforts to void implying a failure of oxygen supply to meet demand. $\mathrm{P}=$ permission to void, $\mathrm{S}=$ uroflow start and $\mathrm{E}=$ uroflow end. (Colors are visible in the online version of the article; http://dx.doi.org/ 10.3233/BSI-140091.)

to occur, and occasionally data match those seen where muscle ischemia develops. In this regard, nonNIRS animal studies have shown a decrease in detrusor blood flow and the onset of hypoxia with partial obstruction of the bladder [69], and also evidence of bladder ischemia [7].

\subsection{Detrusor overactivity and over active bladder}

We and other investigators have studied subjects with symptoms suggesting over active bladder (OAB), and those who have UDS evidence of detrusor overactivity (DO). OAB is a symptom complex with urgency (a sudden compelling desire to pass urine that is difficult to defer), with or without urge incontinence, usually with frequency and nocturia [1]. While two studies suggest NIRS as a diagnostic tool for OAB [39,140], in our experience NIRS changes in these patients are generally not synchronized significantly enough with episodes of DO (as defined by UDS study criteria), or consistent enough in their patterns of change for NIRS to be used for diagnosis [90]. This is probably because both OAB and DO have a number of distinct causal etiologies, including brain lesions, spinal cord lesions, microvascular disease, local bladder pathology, prostate, or lower urinary tract disease. However, in our view the relevance of NIRS in DO and OAB is that published and unpublished data document several distinctive chromophore patterns associated with episodes of bladder overactivity, and involuntary leakage of urine which is the principal symptom of concern to these patients.

These patterns of change suggest that four physiologically distinct events predispose patients with $\mathrm{OAB} / \mathrm{DO}$ to episodes of involuntary urinary leakage:

- Normal (albeit involuntary and untimely) bladder contraction that is likely neurologically mediated;

- A fall in blood volume indicative of a dysfunctional hemodynamic response in the detrusor microcirculation; 
- Oxygen debt/hypoxia, with NIRS changes evident that in voluntary muscle are associated with fatigue during contraction; and,

- The onset of detrusor ischemia (suggested by our data in rare instances).

These findings support there being multiple underlying causal mechanisms for these patients' symptoms. It is also significant that again each of these patterns is comparable to NIRS data obtained in other tissues during specific physiologic events. While supporting the belief that no single NIRS pattern of change would be a robust diagnostic measure in DO or OAB, the data derived via NIRS are relevant, as they identify the probable physiology underlying a patient's symptoms and hence, can guide selection from the therapies available of pharmacologic agents likely to remedy the physiologic and/or functional cause of the symptoms identified via NIRS.

\subsection{Non-neurogenic lower urinary tract dysfunction in children}

We believe the non-invasive optical nature of NIRS makes this a particularly relevant technology for investigating children. There is understandable reluctance to catheterize young patients, and in our experience children readily accept use of a wireless NIRS device which they often compare to a small cell phone. Children also represent a population with significant bladder pathologies, including some unique to younger age groups, such as non-neurogenic voiding dysfunction (NLUTD) [46].

We studied children with NLUTD during natural voiding and published graphs of their data and parallel studies in asymptomatic children [77]. In all asymptomatic children the pattern of change in chromophore concentration was comparable, and matched adult patterns, reflecting full provision by the detrusor microcirculation for the metabolic requirements of normal muscle function [36,111], also, as observed in NIRS studies of voluntary muscle, the increase in $\mathrm{O}_{2} \mathrm{Hb}$ that occurs often exceeds actual oxygen demand [16,41].

Conversely, all children with NLUTD had markedly different patterns of change which showed a predominantly negative trend in $\mathrm{O}_{2} \mathrm{Hb}$ and $\mathrm{tHb}$, in contrast to the positive trend seen in asymptomatic children. The lack of an initial increase in $\mathrm{O}_{2} \mathrm{Hb}$ on initiation of voiding and decrease in $\mathrm{O}_{2} \mathrm{Hb}$ and $\mathrm{tHb}$ during bladder contraction observed, imply a blunting or lack of the hemodynamic response required to provide energy for detrusor contraction. And also the potential for physiologic fatigue to develop during voiding, as happens in striated muscle when exercise intolerance occurs $[41,100,106]$. As children with NLUTD have no known anatomical or neurological basis for their symptoms, these findings suggest that the physiological basis for their voiding dysfunction is a disorder that negatively affects their detrusor hemodynamics and oxygen delivery.

In children, as in adults, we have also observed other patterns of chromophore change during voiding. Published examples include [77]:

- Detrusor blood volume rising and falling repeatedly during voiding;

- Hemodynamic variations followed by a rise in $\mathrm{HHb}$ and a fall in $\mathrm{O}_{2} \mathrm{Hb}$ towards the end of uroflow, implying the late onset of an imbalance in oxygen supply and demand;

- Patterns of changes compatible with the onset of bladder hypoxia in children with particularly severe symptoms.

In these cases hypoxic change is indicated by the 'equal and opposite' trend in $\mathrm{O}_{2} \mathrm{Hb}$ and $\mathrm{HHb}$ that occurs while tHb remains stable (Fig. 9); this pattern matches the characteristic changes in NIRS parameters observed in muscle [130,143] and the rabbit bladder [81] when hypoxia occurs. 


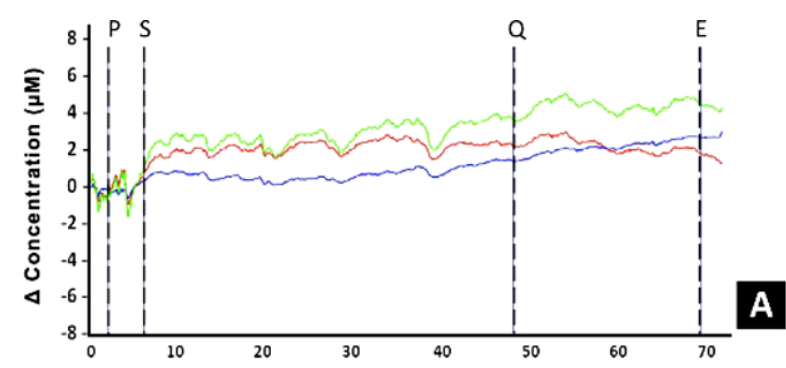

P: Permission to void

S: Uroflow start

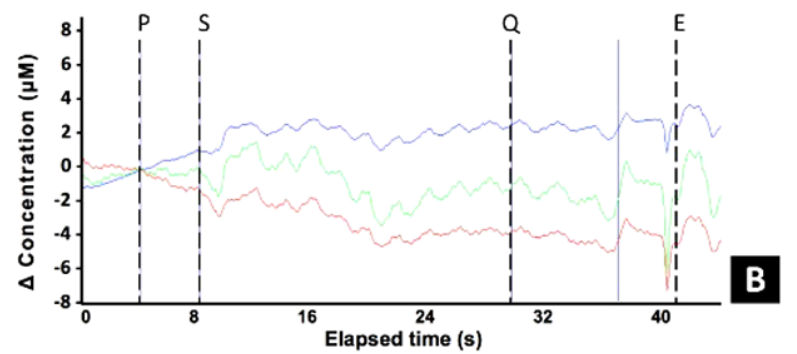

Q: Peak urinflow

E: Uroflow end

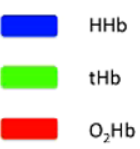

Fig. 9. Two voiding chromophore graphs from children with severe symptoms of dysfunctional voiding. In (A), HHb begins a progressive rise $10 \mathrm{~s}$ prior to maximum flow (Q) which is followed by a sustained fall in $\mathrm{O}_{2} \mathrm{Hb}$. $\mathrm{HHb}$ exceeds $\mathrm{O}_{2} \mathrm{Hb}$ from $60 \mathrm{~s}$ indicating the onset of hypoxia late in voiding. In (B), an 'equal and opposite' rise in $\mathrm{HHb}$ and fall in $\mathrm{O}_{2} \mathrm{Hb}$ begins with intent to void $(\mathrm{P})$. $\mathrm{HHb}$ remains higher than $\mathrm{O}_{2} \mathrm{Hb}$ throughout voiding implying sustained hypoxia in the detrusor. (Colors are visible in the online version of the article; http://dx.doi.org/10.3233/BSI-140091.)

\subsection{Neurogenic bladder}

Spinal cord injury (SCI) results in a wide range of acute and chronic urinary tract complications that adversely affect the health and quality of life of these patients and require frequent clinical assessment [55]. Non-invasive optical monitoring offers the option for these 'at risk' patients to have more assessments than are possible relying on invasive procedures, and provides new insights about the symptoms of urgency and spontaneous leakage of urine common in this population.

We demonstrated the feasibility of using wireless NIRS in pilot studies combining simultaneous NIRS with UDS. Tissue oxygen saturation (TSI \%) was measured in addition to monitoring chromophore change. Interesting data were obtained during bladder filling with a strong consistency seen between $\mathrm{tHb}$ and changes in intravesical pressure across subjects. Generally a positive trend in $\mathrm{O}_{2} \mathrm{Hb} / \mathrm{tHb}$ and TSI $\%$ was seen for the majority of filling, with a fall in all NIRS parameters evident as bladder capacity was approached. However in some patients $\mathrm{HHb}$ and $\mathrm{tHb}$ rose and TSI \% declined following the onset of filling; this implies different physiologic situations exist when the bladder is neurogenic. In addition, where patients experienced involuntary leakage of urine, these leaks were consistently associated with a drop in $\mathrm{O}_{2} \mathrm{Hb} / \mathrm{tHb}$ and TSI \%. These changes were substantial, related to the rise in intravesical pressure and began prior to the leak occurring (Fig. 10).

\subsection{Bladder inflammation}

We have reported how spatially resolved (SR) NIRS can be used to identify an increase in bladder vascularity secondary to inflammatory conditions $[114,116]$. Bacterial cystitis is an infective condition; so SR NIRS was explored as an evaluation measure, because inflammatory changes are known to be associated with an alteration in hemodynamics and oxygenation in other tissues [35]. Children with 


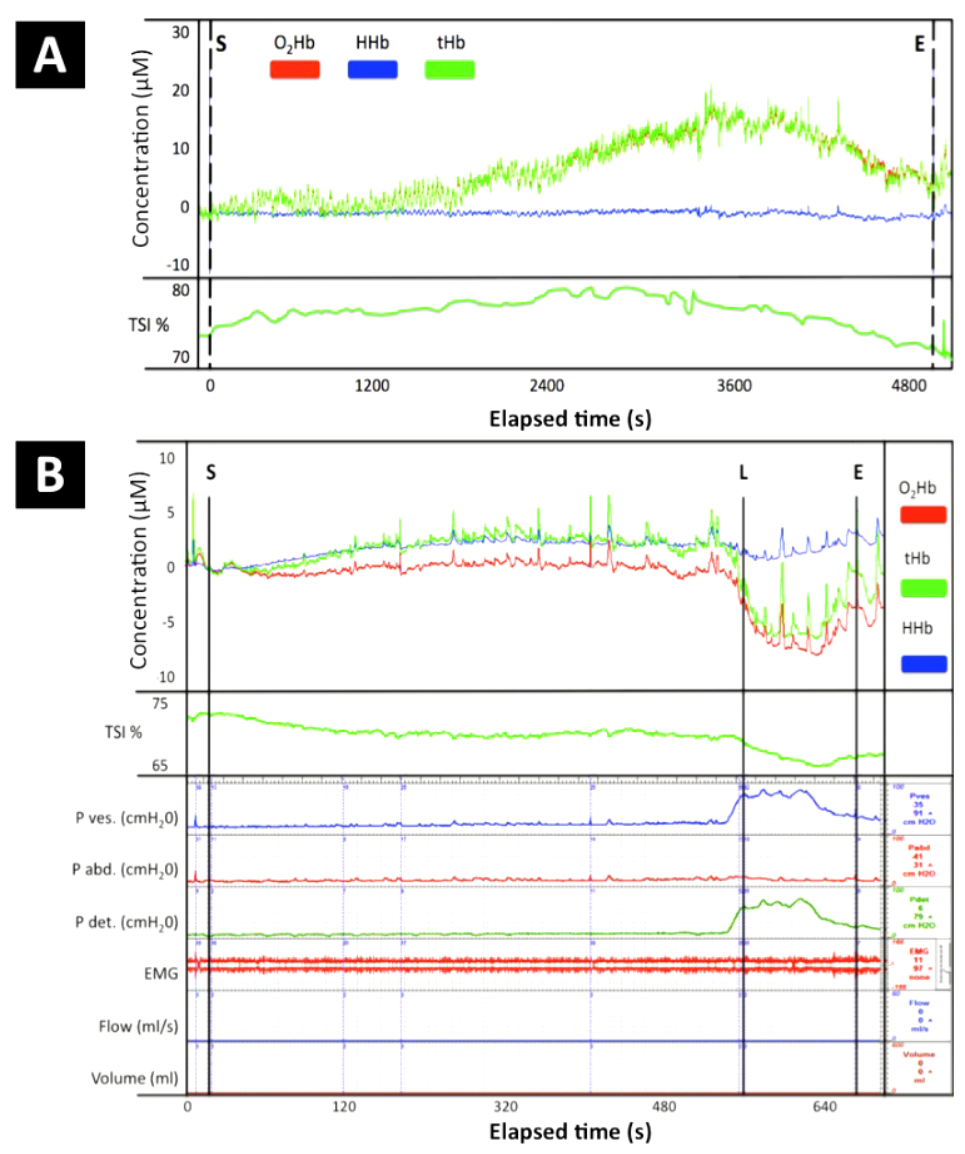

Fig. 10. NIRS data during bladder filling in an asymptomatic male (A), and composite NIRS/UDS data in a paraplegic male with neurogenic bladder (B). In (A), as filling progresses $\mathrm{O}_{2} \mathrm{Hb}$, tHb and TSI \% increase and then fall, while HHB remains unchanged from the start $(\mathrm{S})$ to the end (E) of filling. The point of decrease may represent effective bladder capacity. In (B), throughout filling a progressive rise in HHB and tHb and contemporaneous fall in TSI \% are seen, compatible with oxygen demand exceeding supply. Elevation of vesical and detrusor pressure (P ves./P det.) from 500-640 s is associated with a fall in $\mathrm{tHb} / \mathrm{O}_{2} \mathrm{Hb}$ and TSI \% indicative of marked effects on bladder hemodynamics and oxygenation, and leakage of urine (L). The multiple abrupt unidirectional 'spikes' occurring simultaneously in all 3 NIRS data streams are examples of the effect of movement, and in this case are due to involuntary muscle spasms. (Colors are visible in the online version of the article; http://dx.doi.org/10.3233/BSI-140091.)

symptoms proven to be due to cystitis were compared with a control group who were asymptomatic. TSI $\%$ was measured first over the bladder and then over the quadriceps muscle as a control measure. Detrusor TSI $\%$ values were significantly higher in the UTI group $(p<0.0001)$; quadriceps TSI \% values were not significantly different between groups; however, in those with infection, detrusor values were significantly higher than those in the quadriceps $(p<0.0001)$ [116]. This supports infection causing inflammation of the detrusor muscle, and that an increase in local oxygenation results.

Interstitial cystitis 'Painful bladder syndrome' (IC/PBS), is a symptom complex of urinary urgency, daytime frequency, and suprapubic pain/pressure/discomfort, in the absence of a positive urine culture or another obvious bladder pathology [134,139]. In a pilot study of female subjects with IC/PBS, resting detrusor oxygen saturation (TSI \%) was found to be significantly higher than in control patients with lower urinary tract symptoms due to other bladder conditions $(p<0.0005)$ [114]. This observation 
supports the literature reporting an inflammatory process involving the bladder mucosa as a significant etiology in patients with PBS/IC.

With further studies this technique may offer a screening measure for monitoring the evolution and response to treatment in inflammatory conditions. The use of NIRS at home by patients with SCI is being evaluated; as these patients do not sense many of the symptoms associated with infection, NIRS may help identify when inflammation begins and a urine culture is necessary.

\subsection{Testicular torsion}

Torsion of the testis is an acute urological emergency that requires prompt diagnosis and urgent surgical exploration if testicular function is to be preserved. Torsion results in twisting of the vessels in the spermatic cord that compromises testicular blood flow. Emergent surgical exploration has been considered mandatory, both to confirm the diagnosis and to restore testicular blood flow [60]. Diagnostic modalities to assess testicular perfusion do now offer the potential to select those patients who require surgical treatment; however, most have limitations [113]. Radionuclide imaging, although fairly sensitive, is time consuming and has low specificity; and while color Doppler ultrasonography (CDU) has a high sensitivity and specificity for spermatic cord torsion, intermittent torsion, spontaneous detorsion and atypical cases give false negative results that decrease the reliability and clinical applicability of this method. Detection of twisted sections of the spermatic cord by using high-resolution ultrasonography (HRUS) or MRI has also been suggested as a way to avoid unnecessary operative intervention, but access to these modalities is limited. Recently Burgu has reported the feasibility of using trans-scrotal NIRS measurement of $\mathrm{StO}_{2}$ in the testes in adults to identify the presence of torsion [20], and Shadgan used oxygen saturation measurements (TSI \%) from the right and left spermatic cord to do this in a child [113]. Detection of the difference in oxygen saturation that develops where torsion occludes the blood vessels supplying oxygenated blood to one testis now offers a diagnostic measure in testicular torsion, with the potential to rationalize surgical exploration.

\subsection{Functional NIRS of the bladder}

We have reported the use of a $5 \times 5 \mathrm{~cm}$ two channel four point array to transcutaneously map dynamic change of detrusor hemodynamics during voiding, using 4 channels of a 4 wavelength $\mathrm{CW}$ instrument ('Oxymon' Artinis Medical Systems, BV, The Netherlands) [78]. The array uses the established principles of functional near-infrared spectroscopy (fNIRS) developed for brain mapping, where multi-channel instruments with grids of source-detector pairs are used to detect regional change in oxygenation/hemodynamics [44,58], and video images were generated using incorporated topographic mapping software. The dynamic color video obtained and larger tissue area monitored potentially offer new methodology for investigating regional variations in bladder oxygenation and hemodynamics. The waves of color variation observed in real time across the mapped area suggest regional hemodynamic variations are occurring, providing supportive evidence that the unique coiling/uncoiling mechanism of the bladder vasculature accommodates for changes in the thickness of the bladder wall as the organ fills and empties $[18,73]$. (The video can be viewed on line at: http://link.aip.org/mm/JBOPFO/ 1.3122886/072902jbov1.mov.)

\subsection{Monitoring the urethral sphincter and pelvic floor function}

We have developed a miniaturized NIRS emitter/detector array housed in a customized probe to interrogate the urethral sphincter and the muscles of the pelvic floor trans-vaginally. Both are integral 


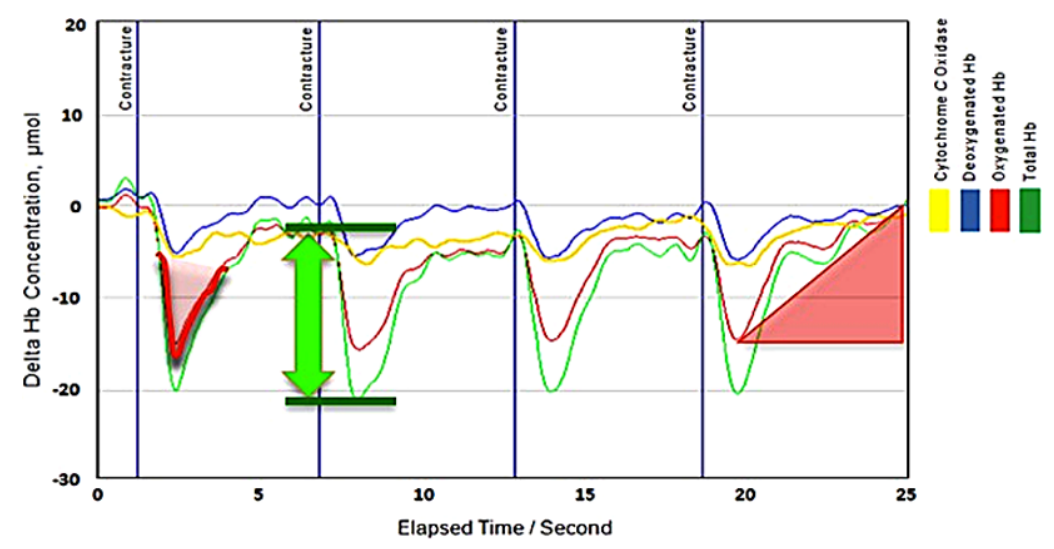

Fig. 11. Chromophore data collected transvaginally showing 4 consecutive sustained pelvic floor contractions. The overlaid points indicate where two measures to quantitate recovery from contraction can be derived - the recovery interval of muscle oxygenation and muscle reoxygenation rate. (Colors are visible in the online version of the article; http://dx.doi.org/10.3233/ BSI-140091.)

elements for continence. The probe utilized 2 emitters placed either side of a single detector to provide 2 channels each with an IOD of $2 \mathrm{~cm}$. Miniature optical cables connect the probe to the standard fiberoptic cables of a commercial CW NIRS instrument via a custom interface. With the probe in position against the anterior vaginal wall, one emitter/detector apposes the bladder detrusor and the other the mid-urethra [118]. In addition to successfully monitoring $\mathrm{O}_{2} \mathrm{Hb}, \mathrm{HHb}$ and $\mathrm{tHb}$ during spontaneous voiding, a series of voluntary pelvic floor contractions were studied using the channel over the mid-urethra. Each contraction had good reproducibility in the patterns and magnitude of chromophore change generated, and an absence of significant movement artifact. An example of one such series of four pelvic floor contractions is shown in Fig. 11.

Two quantitative measures can be derived from such data that are relevant to pelvic floor muscle (PFM) training [124]. PFM is the central component of regimens to restore pelvic floor function after childbirth and address symptoms of involuntary urinary leakage (stress incontinence) which occur [89]. The recovery interval of muscle oxygenation is the time needed for the recovery of $\mathrm{O}_{2} \mathrm{Hb}$ concentration from the maximum level of deoxygenation at the end of contraction to the maximum level of reoxygenation during the post-activity rest period, and reflects the influx of oxygenated arterial blood and continued oxygen utilization during recovery. The muscle reoxygenation rate is calculated as the rate of increase in $\mathrm{O}_{2} \mathrm{Hb}$ during the initial $3 \mathrm{~s}$ immediately after cessation of exercise. This reflects the velocity at which recovery starts after exercise and is directly related to muscle microvascular function [12,24,130,132].

Use of the minimally invasive NIRS assessment technique described to obtain these measurements [89] would allow physicians to individually assess patients, and quantify the efficacy of PFM training regimens pre and post intervention by monitoring quantifiable changes in pelvic floor oxygen supply and demand.

\subsection{Monitoring bladder volume and pressure}

We have reported trials of a prototype device that incorporates a $975 \mathrm{~nm}$ NIR light source [99]. As water has an absorption peak at $975 \mathrm{~nm}$, this device can be used to detect water content in the bladder and to differentiate between an empty bladder, one with low volume, and a full bladder. This will make it possible to detect when the bladder has filled to a defined volume. This is a relevant measure for those 
with neurogenic bladder, as both a quality of life measure to allow elective emptying before accidental incontinence, and as a protective entity where a risk of renal damage due to back pressure exists. This device could also be explored in the context of managing enuresis in children, and loss of continence in the elderly. Loss of continence is the principal medical reason why seniors must leave home and enter residential care.

There is also early evidence that NIRS derived parameters can be modelled mathematically to provide a measure of intravesical pressure. Bladder pressure is of importance to urologists and to date requires direct measurement via a transurethral catheter.

\subsection{Evaluation of effect of pharmacological agents}

Cohorts in therapeutic trial evaluation of alpha blocking agents have also been studied; alpha blockers are a class vasoactive drugs of growing relevance in the treatment of voiding dysfunction [96]. NIRS parameters may well be of value in the evaluation of the effects on the bladder of other pharmaceutical agents currently becoming available for treatment of voiding dysfunction.

\section{The relevance of NIRS to urology}

NIRS is able to provide a range of unique data that reflect physiologic change occurring in the genitourinary system. In particular bladder monitoring now contributes novel physiologic information on changes in detrusor hemodynamics and oxygenation as the bladder fills and empties that add knowledge regarding normal bladder physiology and are relevant in the evaluation of voiding dysfunction $[79,80,82$, $83,102]$. But earlier applications of NIRS in urology remain relevant, including the first, measurement of hemodynamic change in the testis, which is now a legitimate means for clinical detection of testicular torsion.

Although the body of literature on bladder NIRS is still small, it is clear that it is already possible to identify distinctive patterns of change in chromophore concentration in a range of conditions causing voiding dysfunction. Hence, as experience with non-invasive optical monitoring grows, it is probable that distinction will be possible between a larger number of specific bladder pathologies based on the nature of the physiologic changes in oxygenation and hemodynamics evident in each condition. The information that NIRS monitoring can provide is relevant for more comprehensive diagnosis of the underlying cause of bladder problems, and for closer matching of therapy from the medications available to the causal mechanism of the symptoms by basing selection on the site and mode of action of the drug. And, as further studies occur, urologists will identify where and in what conditions NIRS is best utilized. Conducting UDS pressure flow studies with simultaneous bladder NIRS has obvious relevance, and validation of diagnostic algorithms based on chromophore patterns of change may provide a new screening methodology, or non-invasive diagnostic approach to bladder dysfunction of relevance in some contexts. The options for ambulatory and home monitoring using individual or combined elements of the technology are considerable.

\section{Wireless NIRS}

The evolution of wireless NIRS devices and the related computer aided graphics and algorithms demonstrates innovation and clinician/researcher collaboration [64,76,99]. Miniature self-contained de- 
vices with LEDs and SRS capacity make non-invasive transcutaneous bladder monitoring straightforward and an attractive option for clinicians and their patients, including, children, the elderly and those with special needs (e.g. congenital anomalies and spinal cord injury). Wireless systems could be used for home based monitoring selected patients [76,115], and with cell phone transfer of data now possible, review by urologists could happen remotely from the patient. TSI \% measurement of oxygen saturation promises to be a valuable additional component for evaluating bladder function. Advances made with wireless NIRS in the context of bladder studies have contributed significantly to wireless application in other fields $[76,79]$. The devices now available open new possibilities for research and represent an avenue for progress towards other biomedical applications of NIRS.

Current projects include the development of inexpensive miniature wireless devices for use on a large scale to screen for bladder disease in rural clinics in low income countries, with parallel development of software applications and systems to transmit NIRS data using mobile phone networks. A variety of wireless NIRS devices have the potential to be employed in areas of the world where medical resources are scarce. In these environments monitoring, coupled with data transfer and the use of appropriate diagnostic algorithms, could offer screening tests able to identify those requiring preventive treatment to avoid the morbidity associated with advanced, un-treated disease. Such intervention would also rationalize patient referral to limited specialized medical services.

\section{Confidence and reproducibility of bladder NIRS}

The reasons why we have become confident that NIRS monitors the detrusor muscle in the anterior bladder wall, and that the data obtained reflect physiologic change, have been published previously [72, 79,81-83]. Key elements include:

- The physics of penetration, scattering and chromophore absorption of NIR photons;

- The consistent anatomic position of the bladder and ultrasound evidence that the anterior wall maintains its spatial relationship with a NIRS device on the lower abdominal skin during voiding;

- The fact that chromophore changes are only detected over the bladder (not from control emitter/detectors elsewhere on the abdomen), and only occur in direct temporal relationship to events in the voiding cycle;

- That data from bladder studies which indicate changes in detrusor hemodynamics and oxygenation also match NIRS-derived changes evident in physiologic studies in other tissues; and,

- Consistency and reproducibility in patterns and trends of chromophore change is evident in spite of voiding being an event where duration, urine volume, rate of flow, and pressure differ.

Reference to the broader literature has greatly assisted our learning how to interpret bladder NIRS data, and understand how this information reflects and contributes to knowledge of normal bladder physiology and the etiology of lower urinary tract pathology. The relevant literature is broad coming from studies of muscle, brain, and other tissues where reproducible effects on NIRS parameters are seen in response to physiologic effects generated in experimental studies, or observed to occur in the microcirculation due to specific systemic and organ pathology [12,13,16,24,34,42,53,59,100,130,132,133,142]. Reproducibility of data in asymptomatic adults and children has been illustrated [77], and also between NIRS devices [83]. Many systemic pathologies affecting the vasculature of the human body are known to compromise the function of the detrusor, and we suggest these adversely impact bladder function by compromising the ability of the microcirculation to respond normally to an increase in oxygen demand during bladder contraction. 
Our data also indicate that a number of local bladder pathologies, such as bladder outlet obstruction, generate organ-specific changes in the detrusor muscle that adversely affect blood flow, muscle metabolism, and contractility. Importantly, several NIRS-derived patterns of change indicative of impaired hemodynamics and/or oxygenation that occur in symptomatic voiding dysfunction are seen consistently.

We conducted animal experiments to investigate the potential impact of subcutaneous tissue on bladder monitoring by comparing chromophore changes occurring in response to induced hypoxia during transcutaneous monitoring, and when optodes were placed directly on the wall of the surgically exposed bladder. An appropriate IOD was used in each circumstance to account for the different depth of light penetration required. The patterns of change from both optode locations were the same, and matched those seen in other tissues in response to hypoxia [81]. Additional corroboration of the physiologic relevance of bladder NIRS data came when the organ's blood supply was clamped, as changes indicative of ischemia occurred immediately.

Legitimate concerns have been expressed over what effect movement [38] may have on bladder spectroscopy, whether change in bladder size as the organ contracts compromises data, and if NIR photons penetrate as far as the anterior bladder wall. With spontaneous voluntary movements and involuntary muscle spasm (in paraplegic patients) the effect is readily evident as abrupt simultaneous unidirectional changes ('spikes') of short duration in the magnitude of $\mathrm{O}_{2} \mathrm{Hb}, \mathrm{HHb}$ and $\mathrm{tHb}$ (e.g. in graph B, Fig. 10) $[79,82,125]$. Similarly, during simultaneous NIRS and UDS studies, when abdominal contraction occurs during strenuous voiding, these same abrupt changes in all NIRS parameters are seen in conjunction with an obvious rise in abdominal pressure and increased electromyogram activity. However, over all, even in the presence of movement, the important physiologic trends in NIRS data remain evident, as can be seen when comparing muscle data at rest and during active exercise (e.g. in graph B, Fig. 12) $[40,82,83]$.

Ultrasound data [126] of how the position and dimensions of the bladder alter during phases of the voiding cycle suggest minimal interference to monitoring by organ movement during voiding. The monitoring site on the abdominal skin appears optimal as change in bladder size predominantly affects the dome rather than the base, and the relationship between the NIRS sensor and the anterior wall of the bladder remains relatively constant during voiding. The reproducibility and degree of discriminant ability of NIRS-derived 'diagnostic' algorithms would also be unlikely if change in bladder size or other artefact contributed to any meaningful degree to bladder NIRS data $[40,85,121,129,144,145]$.

Two other observations specifically support bladder spectroscopy providing physiologic data uncontaminated by movement. Significant hemodynamic change (increase in $\mathrm{tHb} / \mathrm{O}_{2} \mathrm{Hb}$ ) is evident immediately before voiding begins, i.e. while the bladder volume remains constant and no change in bladder size has occurred. We have also documented significant variations in the concentration and trends of $\mathrm{O}_{2} \mathrm{Hb}, \mathrm{HHb}$ and $\mathrm{tHb}$ in subjects with voiding delay that are individually indicative of changes in blood volume, an increase in the availability of oxygen, and increased oxygen consumption when no urine has been passed [72,83]. Published graphs indicate that in these subjects, in spite of the decision and ongoing intent to void the physiologic changes evident via NIRS occur when there is no alteration in the volume of urine in the bladder or change in the organ's size or position [83].

Consequently it is more probable than not that the ability of NIRS to provide monitoring data of value during evaluation of patients with voiding dysfunction is not negatively impacted by either movement or change in bladder size. 


\section{Future prospects}

As experience with bladder NIRS grows, it is probable that distinction will be evident between a broad range of specific bladder pathologies, based on the nature of the physiologic effects generated in each condition on oxygenation and hemodynamics. This is already possible in several groups of patients of both genders from childhood through to an advanced age $[77,79,80,83]$. There is also real potential for NIRS to be used alone as a screening tool for the initial evaluation of voiding disorders, for monitoring tissue oxygenation changes, investigating bladder filling, or as an independent diagnostic entity. However, even if NIRS were only to be used in conjunction with simultaneous conventional UDS pressure flow monitoring studies, the additional physiologic information available to clinicians would probably contribute to better understanding of causal pathology in patients with voiding dysfunction, aid logical choice of therapeutic agents, and provide a measure of treatment efficacy.

In the future, bladder pathologies that have not yet been studied using NIRS will be investigated noninvasively. Greater use of devices with SR-NIRS capacity will enable the relevance of absolute changes in bladder wall oxygen saturation to be explored further, and will likely offer significant opportunities for monitoring the onset of acute disease, as well as trends in disease evolution and recovery of bladder function following treatment. Incorporation of wavelengths that optimize detection of NIR photon attenuation by water will enable new monitoring of changes in bladder size that are relevant to a range of pathologies, and age groups. Involuntary voiding in children (enuresis) and in the elderly lend themselves to monitoring with a small NIRS device containing an alarm that activates when the bladder is

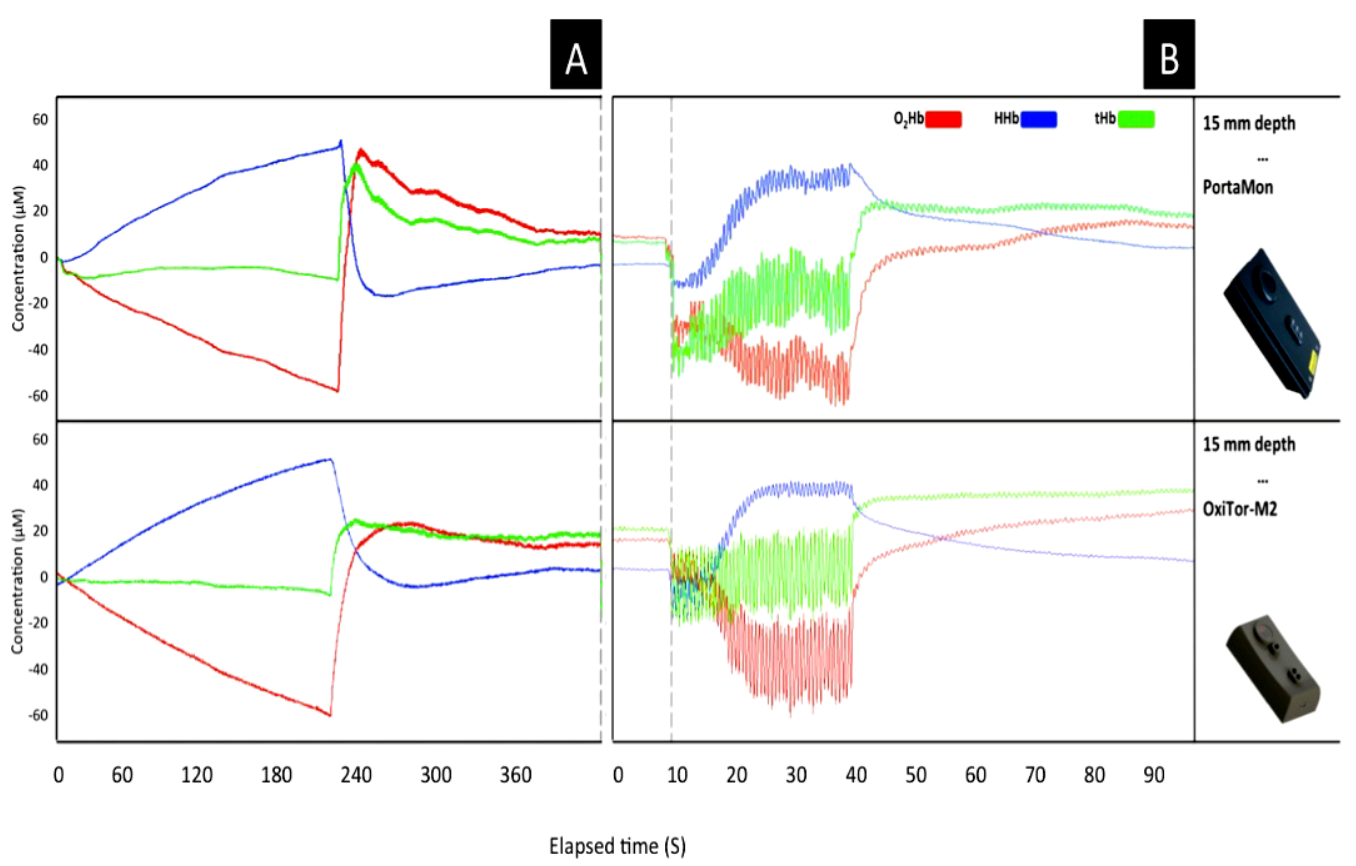

Fig. 12. Sequential graphs showing the consistency of wireless NIRS data during (A) 4 min of tourniquet induced forearm ischemia with 3 min recovery, followed by (B) $30 \mathrm{~s}$ of isotonic exercise and recovery, monitored by two different wireless NIRS devices in one subject. In graph (B) the trend direction and overall magnitude of chromophore change remain evident and reproducible between devices in the presence of movement generated during the period of exercise (10-40 s). (Colors are visible in the online version of the article; http://dx.doi.org/10.3233/BSI-140091.) 
full, as do conditions such a neurogenic bladder, where unrecognized over-distention of the bladder can result in involuntary urinary leakage, or over time lead to back pressure damage to the kidney.

Device miniaturization will continue to expand the scope and applications of NIRS in urology, and for bladder spectroscopy in particular. CAD and related software programs will improve the design and matching of device specifications to clinicians' needs, contribute to ease of use of devices, and ensure that they precisely fulfill the intent of their design. Devices with LED's will become inexpensive, and software development will occur that improves the quality, storage, display and transmission of data. Custom algorithms will contribute new and improved diagnostic methodology, and add refinements that could, for example, provide a measure reflecting bladder pressure from chromophore data in the case of bladder studies, or attenuate movement artefact in situations where this would improve the information derived via NIRS.

NIRS screening of patients in resource-poor environments for treatable pathologies will occur. This will identify those who with early treatment can be spared the morbidity, poor quality of life and premature mortality associated with late recognition of defined diseases. An example already being explored is early recognition of bladder outlet obstruction in low income countries, where NIRS screening in clinics in rural areas combined with remote transfer of data and use of diagnostic algorithms will make early diagnosis possible, allow provision of drug therapy to reduce morbidity, and rationalize referral to scarce resources able to provide advanced levels of care.

To date the use of NIRS to evaluate the effect of bladder pharmaceuticals has only involved alpha blockers; but as newer therapeutic agents come on line, including those with recognized effects on the bladder microvasculature, NIRS should contribute information relevant to the site and mode of action, or efficacy of these drugs.

Whether NIRS comes to be used in urology for research, clinical study, home monitoring, screening programs, or diagnosis is for basic scientists and urologists to determine through further collaborative studies. However, from the research done to date there are strong grounds to undertake the due diligence required to establish where NIRS can and cannot contribute. In urology, as in other fields and with any biomedical applications, it is of particular importance in this process to use knowledge acquired from NIRS studies by researchers in other disciplines. The physiologic insights learned through NIRS research into muscle and brain function, the contributions of NIRS in exercise science, and the established clinical relevance of the scientific principles of NIRS in oximetry are key examples of why such knowledge should be translated across disciplines, and confidence drawn to explore clinical diagnostic opportunities. NIRS is often spoken of in the context of 'having potential'. But in urology bladder spectroscopy in particular now represents a disruptive technology that can add unique physiologic insights to diagnostic evaluation. Hence NIRS can be of benefit to a significant population worldwide if appropriate studies are undertaken, and the relevant clinical experience sought to optimize use of this technology.

\section{Acknowledgements}

This work was only possible because of inspiration from early career mentors, collaborative work with many talented individuals, and funding from leading agencies. Osmund Reynolds taught me to translate clinical issues into research questions and generously encouraged me to share his interest in NIRS; I learned the importance of scientific rigor from Leonard Strang, and the central place of good communication in research and clinical medicine from Sir Robert Boyd. David Delpy and his colleagues at UCL and Hamamatsu Photonics were always helpful as my team introduced brain and spinal cord NIRS 
into Canada, with monitoring during cardiac and neurosurgery and in the newborn nursery, and Willy Colier at Artinis Medical gave invaluable support over instruments and design as we embarked on applications in urology. Roy Gagnon was meticulous with data collection. Lynn Stothers is the source of all my urologic knowledge and the relevance of NIRS for studying voiding dysfunction. Young investigators such as Manicum Moodley and Babak Shadgan have consistently advanced my work with searching questions and profound insights. Recently, the vision of Grand Challenges Canada has enabled bladder NIRS to be explored as a means of reducing the burden of disease in the developing world. And like all investigators, I am indebted to leading journal editors and their peer reviewers for the publication of my work.

\section{References}

[1] P.H. Abrams, L. Cardozo, M. Fall, D. Griffiths, P. Rosier, U.L. Ulmsten, P. van Kerrebroeck, A. Victor and A. Wein, The standardisation of terminology in lower urinary tract function: report from the standardisation sub-committee of the International Continence Society, Urology 61(1) (2003), 37-49.

[2] P.H. Abrams and D.H. Griffiths, The assessment of prostatic obstruction from urodynamic measurements and from residual urine, Br. J. Urol. 51 (1979), 129-134.

[3] A. Amelink, D.J. Kok, H.J.C.M. Sterenborg and J.R. Scheepe, In vivo measurement of bladder wall oxygen saturation using optical spectroscopy, J. Biophotonics 4(10) (2011), 715-720.

[4] K.-E. Andersson and A. Arner, Urinary bladder contraction and relaxation: Physiology and pathophysiology, Physiol. Rev. 84(3) (2004), 935-986.

[5] R.N. Aslin and J. Mehler, Near-infrared spectroscopy for functional studies of brain activity in human infants: promise, prospects, and challenges, J. Biomed. Opt. 10(1) (2005), 11009.

[6] O. Aydogdu, B. Burgu, P.U. Gocun, E. Ozden, O. Yaman, T. Soygur, A. Dursun and K. Aydor, Near infrared spectroscopy to diagnose experimental testicular torsion: comparison with Doppler ultrasound and immunohistochemical correlation of tissue oxygenation and viability, J. Urol. 187(2) (2012), 744-750.

[7] K.M. Azadzoi, T. Tarcan, R. Kozlowski, R.J. Krane and M.B. Siroky, Overactivity and structural changes in the chronically ischemic bladder, J. Urol. 162(5) (1999), 1768-1778.

[8] K.M. Azadzoi, S.V. Yalla and M.B. Siroky, Oxidative stress and neurodegeneration in the ischemic overactive bladder, J. Urol. 178(2) (2007), 710-715.

[9] J.R. Baena and B. Lendl, Raman spectroscopy in chemical bioanalysis, Curr. Opin. Chem. Biol. 8 (2004), 534-539.

[10] A. Belenky, Y. Abarbanel, M. Cohen, O. Yossepowitch, P.M. Livne and G.N. Bachar, Detrusor resistive index evaluated by Doppler ultrasonography as a potential indicator of bladder outlet obstruction, Urology 62(4) (2003), 647-650.

[11] E.E. Benarroch, Neural control of the bladder: Recent advances and neurologic implications, Neurology 75 (2010), 1839-1846.

[12] Y.N. Bhambhani, Muscle oxygenation trends during dynamic exercise measured by near-infrared spectroscopy, Can. J. Appl. Physiol. 29 (2004), 504-523.

[13] Y.N. Bhambhani, Review: Application of near infrared spectroscopy in evaluating cerebral and muscle haemodynamics during exercise and sport, J. NIRS 20(1) (2012), 117-139.

[14] B.F. Blok, A.T. Willemsen and G. Holstege, A PET study on brain control of micturition in humans, Brain 120(1) (1997), $111-121$.

[15] G. Bottiroli and A.C. Croce, Autofluorescence spectroscopy of cells and tissues as a tool for biomedical diagnosis, Photochem. Photobiol. Sci. 3 (2004), 189-201.

[16] R. Boushel, H. Langberg, J. Olesen, J. Gonzales-Alonzo, J. Bulow and M. Kjaer, Monitoring tissue oxygen availability with near infrared spectroscopy (NIRS) in health and disease, Scand. J. Med. Sci. Sports 11 (2001), 213-222.

[17] A. Bozkurt, A. Rosen, H. Rosen and B. Onaral, A portable near infrared spectroscopy system for bedside monitoring of newborn brain, Biomedical Engineering Online 4 (2005), 29.

[18] A.F. Brading, J.E. Greenland, I.W. Mills, G. McMurray and S. Symes, Blood supply to the bladder during filling, Scand. J. Urol. Nephrol. Suppl. 201 (1999), 25-31.

[19] L. Breiman, J.H. Friedman, R.A. Olshen and C.J. Stone, Classification and Regression Trees, CRC Press, New York, 1998.

[20] B. Burgu, O. Aydogdu, R. Huang, T. Sogur, O. Yaman and L. Baker, Pilot feasibility study of transscrotal near infrared spectroscopy in the evaluation of adult acute scrotum, J. Urol. 190(1) (2013), 124-129. 
[21] A.L. Burnett, R.P. Allen, D.M. Davis, D.C. Wright, I.N. Trueheart and B. Chance, Near infrared spectrophotometry for the diagnosis of vasculogenic erectile dysfunction, Int. J. Impot. Res. 12 (2000), 247-254.

[22] M. Canpolat, S. Yucel, A. Sircan-Kucuksayan, A. Kol, H. Ogzur Kazanci and T. Denkceken, Diagnosis of testicular torsion by measuring attenuation of dual wavelengths in transmission geometry across the testis, an experimental study in a rat model, Urology 79 (2012), 966e9-996.e12.

[23] G.A. Capraro, T.J. Mader, B.F. Coughlin, C. Lovewell, M.R. St. Louis, M. Tirabassi, G. Wadie and H.A. Smithline, Feasibility of using near-infrared spectroscopy to diagnose testicular torsion: an experimental study in sheep, Ann. Emerg. Med. 49 (2007), 520-525.

[24] B. Chance, M.T. Dait, C. Zhang, T. Hamaoka and F. Hagerman, Recovery from exercise-induced desaturation in the quadriceps muscles of elite competitive rowers, Am. J. Physiol. 262 (3 Pt. 1) (1992), C766-C775.

[25] B.I. Chung, G. Sommer and J.D. Brooks, Anatomy of the lower urinary tract and male genitalia, in: Wein: CampbellWalsh Urology, A.J. Wein, L.R. Kavoussi, A.C. Novick, A.W. Partin and C.A. Peters, eds, 10th edn, Saunders, 2011, pp. 33-70.

[26] W.M. Colier, F.M. Froeling, J.D. de Vries and B. Oesburg, Measurement of the blood supply to the abdominal testis by means of near infrared spectroscopy, Eur. Urol. 27 (1995), 60-66.

[27] D. Contini, 1. Zucchelli, L. Spinelli, M. Caffini, R. Re, A. Pifferi, R. Cubeddu and A. Torricelli, Review: Brain and muscle near infrared spectroscopy/imaging techniques, J. NIRS 20(1) (2012), 15-27.

[28] C.E. Cooper and R. Springett, Measurement of cytochrome oxidase and mitochondrial energetics by near-infrared spectroscopy, Phil. Trans. R. Soc. Lond. B Biol. Sci. 352(1354) (1997), 669-676.

[29] M. Cope, D.T. Delpy, S. Wray, J.S. Wyatt and E.O.R. Reynolds, A CCD spectrometer to quantitate the concentration of chromophores in living tissue utilizing the absorption peak of water at $975 \mathrm{~nm}$, Adv. Exp. Med. Biol. 248 (1989), 33-40.

[30] J. Davignon and P. Ganz, Role of endothelial dysfunction in atherosclerosis, Circulation 109(23 Suppl 1) (2004), III27III32.

[31] D.T. Delpy and M. Cope, Quantification in tissue near-infrared spectroscopy, Phil. Tans. R. Soc. Lond. B Biol. Sci. 352(1354) (1997), 649-659.

[32] D.T. Delpy, M. Cope, P. van der Zee, S. Arridge, S. Wray and J.S. Wyatt, Estimation of optical path length through tissue from direct time of flight measurements, Phys. Med. Biol. 33 (1988), 1433-1442.

[33] E. Dugan, S.J. Cohen, D. Robinson, R. Anderson, J. Preisser, P. Suggs, K. Pearce, U. Poehling and P. McGann, The quality of life of older adults with urinary incontinence: determining generic and condition specific predictors, Qual. Life Res. 7 (1998), 337-344.

[34] T. Durduran, Y. Guoqiang, Z. Chao, G. Lech, B. Chance and A.G. Yodh, Quantification of muscle oxygenation and flow of healthy volunteers during cuff occlusion of arm and leg flexor muscles and plantar flexion exercise, Proc. SPIE 4955(1) (2003), 447-453.

[35] R.M. Echols, R.L. Tosiello, D.C. Haverstock and A.D. Tice, Demographic, clinical, and treatment parameters influencing the outcome of acute cystitis, Clin. Infect. Dis. 29 (1999), 113-119.

[36] C.G. Ellis, J. Jagger and M. Sharpe, The microcirculation as a functional system, Crit. Care 9(Suppl 4) (2005), S3-S8.

[37] C.E. Elwell and C.E. Cooper, Making light work: illuminating the future of biomedical optics, Phil. Trans. R. Soc. A 369 (2011), 4358-4379.

[38] F.F. Farag and J.P. Heesakkers, Non-invasive techniques in the diagnosis of bladder storage disorders, Neurourol. Urodyn. 30 (2011), 1422-1428.

[39] F.F. Farag, F.M. Martens, K.W. D’Hauwers, W.F. Feitz and J.P. Heesakkers, Near-infrared spectroscopy: A novel, noninvasive, diagnostic method for detrusor over activity in patients with overactive bladder symptoms - A preliminary and experimental study, Eur. Urol. 59(5) (2011), 752-762.

[40] F.F. Farag, J. Meletiadis, M.D. Saleem, W.F. Feitz and F.P. Heesakkers, Near-infrared spectroscopy of the urinary bladder during voiding in men with lower urinary tract symptoms: A preliminary study, BioMed. Research International 2013 (2013), Article ID 452857, 7 pp.

[41] M. Ferrari, T. Binzoni and V. Quaresima, Oxidative metabolism in muscle, Phil. Trans. R. Soc. Lond. B 352 (1997), 677-683.

[42] M. Ferrari, L. Mottola and V. Quaresima, Principles, techniques and imitations of near infrared spectroscopy, Can. J. Appl. Physiol. 29 (2004), 463-487.

[43] M. Ferrari, M. Muthalib and V. Quaresima, The use of near-infrared spectroscopy in understanding skeletal muscle physiology: recent developments, Phil. Trans. R. Soc. A 369(1955) (2011), 4577-4590.

[44] M. Ferrari and V. Quaresima, Review: Near infrared brain and muscle oximetry: from the discovery to current applications, J. NIRS 20(1) (2012), 1-14.

[45] C.J. Fowler and D.J. Griffiths, A decade of functional brain imaging applied to bladder control, Neurourol. Urodyn. 29 (2010), 49-55.

[46] X. Game, E. Chartier-Kastler and A. Ruffion, Lower urinary tract dysfunction and non-neurogenic neurogenic bladder or 'dysfunctional voiding', Progress en Urologie 17(3) (2007), 406-414. 
[47] J.A. Gosling, J.S. Dixon and R.G. Lendon, The autonomic innervation of the human male and female bladder neck and proximal urethra, J. Urol. 118(2) (1977), 302-307.

[48] D. Griffiths, Basics of pressure-flow studies, World J. Urol. 13 (1995), 30-33.

[49] D. Griffiths, Imaging bladder sensations, Neurourol. Urodyn. 26(S6) (2007), 899-903.

[50] A. Groutz and J.G. Blavis, Non-neurogenic female voiding dysfunction, Curr. Opin. Urol. 12(4) (2002), 311-316.

[51] R. Guevara, L. Stothers and A.J. Macnab, Mathematical modeling methodology for generation of a diagnostic algorithm using near-infrared data, Spectroscopy 25(1) (2011), 1-11.

[52] B. Hallacoglu, H.J. Paltiel, P. Garfollo, A. Alomari, A. Sassarola, S. Fantini, G. Cannon, H. Padua and R.S. Matulewicz, Noninvasive assessment of testicular torsion in rabbits using frequency-domain near-infrared spectroscopy: prospects for pediatric urology, J. Biomed. Opt. 14 (2009), 054027, doi:10.1117/1.3253318.

[53] T. Hamaoka, K.K. McCully, M. Niwayama and B. Chance, The use of muscle near-infrared spectroscopy in sport, health and medical sciences: recent developments, Phil. Trans. R. Soc. A 369 (2011), 4591-4601.

[54] D.N. Harris, F.M. Cowans, D.A. Wertheim and S. Hamid, NIRS in adults - effects of increasing optode separation, Adv. Exp. Med. Biol. 345 (1994), 837-840.

[55] B.L. Hicken, D. Putzke and J.S. Richards, Bladder management and quality of life after spinal cord injury, Am. J. Phys. Med. Rehabil. 80 (2001), 916-922.

[56] G. Hohlbrugger, F. Frauscher, H. Strasser, A. Stenzl and G. Bartsch, Evidence for the autoregulation of vesical circulation by intravesical potassium chloride and distension in the normal human bladder, BJU Int. 85(4) (2000), 412-415.

[57] S. Homma, H. Eda, S. Ogasawara and A. Kagaya, Near-infrared estimation of $\mathrm{O}_{2}$ supply and consumption in forearm muscles working at varying intensity, J. Appl. Physiol. 80(4) (1996), 1279-1284.

[58] Y. Hoshi, Functional near-infrared spectroscopy: current status and future prospects, J. Biomed. Opt. 12(6) (2007), 062106.

[59] F.F. Jobsis, Noninvasive, infrared monitoring of cerebral and myocardial oxygen sufficiency and circulatory parameters, Science 198(4323) (1977), 1264-1267.

[60] S. Kapoor, Testicular torsion: A race against time, Int. J. Clin. Pract. 62(5) (2008), 821-827.

[61] R.W. Katzberg, Urography in the 21 st century: New contrast media, renal handling, imaging characteristics, and nephrotoxicity, Radiology 204 (1997), 297-312.

[62] G.J. Kemp, A.V. Crowe, H.K. Anijeet, Q.Y. Gong, W.E. Bimson, S.P. Frostick, J.M. Bone, G.M. Bell and J.N. Roberts, Abnormal mitochondrial function and muscle wasting, but normal contractile efficiency, in haemodialysed patients studied non-invasively in vivo, Nephrol. Dialysis Transplan. 19 (2004), 1520-1527.

[63] R.T. Kershen, K.M. Azadzoi and M.B. Siroky, Blood flow, pressure and compliance in the male human bladder, J. Urol. 168(1) (2002), 121-125.

[64] C.K. Kim, S. Lee, D. Koh and B.M. Kim, Development of a wireless NIRS system with dynamic removal of motion artefacts, Biomedical Engineering Letters 1(4) (2011), 254-259.

[65] R. Kozlowski, M.B. Siroky, R.J. Krane and K.M. Azadzoi, Regulation of blood flow and microcirculation resistance in rabbit bladder, J. Urol. 168 (2002), 1608-1614.

[66] W. Krause, P. Muschick and U. Kruger, Use of near-infrared reflection spectroscopy to study the effects of X-ray contrast media on renal tolerance in rats: effects of a prostacyclin analogue and of phosphodiesterase inhibitors, Invest. Radiol. 37 (2002), 698-705.

[67] V. Kupelian, J.T. Wei, M.P. O’Leary, J.W. Kusek, H.J. Litman, C.J. Link, J.B. McKinlay and BACH Survey Investigators, Prevalence of lower urinary tract symptoms and effect on quality of life in a racially and ethnically diverse random sample, The Boston Areas Community Health (BACH) survey, Arch. Intern. Med. 166(21) (2006), 2381-2387.

[68] P. Latimer, Absolute adsorption and scattering spectrophotometry, Arch. Biochem. Biophys. 119(1) (1997), 580-581.

[69] R.M. Levin, L.J. O'Connor, R.J. Leggett, C. Whitebeck and P. Chichester, Focal hypoxia of the obstructed rabbit bladder wall correlates with intermediate decompensation, Neurourol. Urodynam. 22 (2003), 156-163.

[70] A.T.L. Lin, M.T. Chen, C.H. Yang and L.S. Chang, Blood flow of the urinary bladder: effects of outlet obstruction and correlation with energetic metabolism, Neurol. Urodyn. 14 (1995), 285-292.

[71] W.Y. Loh, Classification and regression tree methods, in: Encyclopedia of Statistics in Purity and Reliability, F. Ruggeri, R.S. Kenett and F.W. Faltin, eds, Wiley, London, 2008, pp. 315-323.

[72] A.J. Macnab, Applications of near infrared spectroscopy in urology, in: Infrared Spectroscopy: Theory, Development and Applications, D. Cozzolino, ed., Nova Science Publishers Inc., New York, 2014, pp. 127-168.

[73] A.J. Macnab, B. Friedman, B. Shadgan and L. Stothers, Bladder anatomy physiology and pathophysiology: elements that suit Near Infrared Spectroscopic evaluation of voiding dysfunction, Biomedical Spectroscopy and Imaging 1(3) (2012), 223-235.

[74] A.J. Macnab, R.E. Gagnon, F.A. Gagnon and J.G. LeBlanc, NIRS monitoring of brain and spinal cord - detection of adverse intraoperative events, Spectroscopy 17(2,3) (2003), 483-490.

[75] A.J. Macnab, R.E. Gagnon and L. Stothers, Clinical NIRS of the urinary bladder: A demonstration case report, Spectroscopy 19(4) (2005), 207-212. 
[76] A.J. Macnab and B. Shadgan, Biomedical applications of wireless continuous wave near infrared spectroscopy, Biomedical Spectroscopy and Imaging 1(3) (2012), 205-222.

[77] A.J. Macnab, B. Shadgan, K. Afshar and L. Stothers, Near-Infrared Spectroscopy of the bladder: New parameters for evaluating voiding dysfunction, Int. J. Spect. 2011 (2011), Article ID 814179.

[78] A.J. Macnab, B. Shadgan and L. Stothers, Functional near infrared spectroscopy (fNIRS); dynamic topography of the human bladder during voiding, J. Biomed. Optics 14(2) (2009), 020507.

[79] A.J. Macnab, B. Shadgan and L. Stothers, The evolution of wireless near infrared spectroscopy: applications in urology and rationale for clinical use, J. NIRS 20 (2012), 57-73.

[80] A.J. Macnab, B. Shadgan and L. Stothers, Monitoring detrusor oxygenation and hemodynamics non-invasively during dysfunctional voiding, Advances in Urology 2012 (2012), Article ID 676303.

[81] A.J. Macnab, B. Shadgan and L. Stothers, Validation of transcutaneous NIRS monitoring of bladder hemodynamics and oxygenation using a rabbit model, Biomedical Spectroscopy and Imaging 2(2) (2013), 91-99.

[82] A.J. Macnab, B. Shadgan and L. Stothers, Monitoring physiologic change in the bladder in health and disease. A new biomedical application of near-infrared spectroscopy, Biomedical Spectroscopy and Imaging 2(4) (2013), 289-299.

[83] A.J. Macnab, B. Shadgan, L. Stothers and K. Afshar, Ambulant monitoring of bladder oxygenation and hemodynamics using wireless near-infrared spectroscopy, Can. Urol. Assoc. J. 7(1,2) (2013), E98-E104.

[84] A.J. Macnab and L. Stothers, Receiver operator curves describing near infrared spectroscopy (NIRS) changes during pressure flow studies in men with obstruction, J. Urol. 177(4) (2007), 502-503.

[85] A.J. Macnab and L. Stothers, Near infrared spectroscopy (NIRS): Validation of bladder-outlet obstruction assessment using non-invasive parameters, Can. J. Urol. 15(5) (2008), 4241-4248.

[86] A.J. Macnab and L. Stothers, Development of a near infrared spectroscopy instrument for applications in urology, Can. J. Urol. 15(5) (2008), 4233-4240.

[87] S. Madersbacher, A. Pycha, G. Schatzl, C. Mian, C.H. Klinger and M. Marberger, The aging lower urinary tract: a comparative urodynamic study of men and women, Urology 51(2) (1998), 206-212.

[88] D.A. Madsen, Engineering Drawing \& Design, Delmar, Clifton Park, NY, 2012. ISBN 1111309574.

[89] A. Marques, L. Stothers and A.J. Macnab, The status of pelvic floor muscle training for women, Can. Urol. Assoc. J. 4(6) (2010), 419-424.

[90] H. Mastoroudes, I. Giarenis, M. Vella, S. Srikrishna, D. Robinson, L. Cardozo, I. Karrouze, A. Campbell and A. Macnab, Use of near infrared spectroscopy as an alternative to videourodynamics to detect detrusor overactivity in women with the overactive bladder syndrome, Urology 80(3) (2012), 547-550.

[91] S. Matcher, P. Kirkpatrick, K. Nahid, M. Cope and D.T. Delpy, Absolute quantification methods in tissue near infrared spectroscopy, Proc. SPIE 2389 (1995), 486-495.

[92] N. Matsumoto, S. Ichimura, T. Hamaoka, T. Osada, M. Hattori and S. Miyakawa, Impaired muscle oxygen metabolism in uremic children: improved after renal transplantation, Am. J. Kidney Dis. 48(3) (2006), 473-480.

[93] S. Matsumoto, A. Ishikawa, H. Kume, T. Takeuchi and Y. Homma, Near infrared spectroscopy study of the central nervous activity during artificial changes in bladder sensation in men, Int. J. Urol. 16(9) (2009), 760-764.

[94] K. Matsushita, S. Homma and E. Okada, Influence of adipose tissue on muscle oxygenation measurement with NIRS instrument, Proc. SPIE 3194 (1998), 151-165.

[95] E.J. Meuleman and W.L. Diemont, Investigation of erectile dysfunction: diagnostic testing for vascular factors in erectile dysfunction, Urologic Clinics of North America 22(4) (1995), 803-819.

[96] M.C. Michel, The forefront for novel therapeutic agents based on the pathophysiology of lower urinary tract dysfunction: $\alpha$-blockers in the treatment of male voiding dysfunction - How do they work and why do they differ in tolerability?, J. Pharmacol. Sci. 112 (2010), 151-157.

[97] A.J. Miodonski and J.A. Litwin, Microvascular architecture of the human urinary bladder wall: a corrosion casting study, Anat. Rec. 254(3) (1999), 375-381.

[98] B. Molavi, G. Dumont, B. Shadgan and A. Macnab, Attenuation of motion artifact in near infrared spectroscopy signals using a wavelet based method, Proc. SPIE 7890 (2011), 78900M, doi:10.1117/12.

[99] B. Molavi, B. Shadgan, A.J. Macnab and G. Dumont, Non-invasive optical monitoring of bladder filling to capacity using a wireless NIRS device, IEEE Transactions in Biomedical Circuits and Systems 8(3) (2014), 325-333.

[100] G. Murthy, A.R. Hargens, S. Lehman and D.M. Rempel, Ischemia causes muscle fatigue, J. Ortho. Res. 19(3) (2001), 436-440.

[101] V. Nitti, Pressure flow urodynamic studies: the gold standard for diagnosing bladder outlet obstruction, Reviews in Urology 7(Suppl. 6) (2005), S14-S21.

[102] J. Pannek, Editorial comment on: Classification of male lower urinary tract symptoms using mathematical modelling and a regression tree algorithm of noninvasive near-infrared spectroscopy parameters, Euro Urol. 57 (2010), 332-333.

[103] A. Petrova and R. Mehta, Near-infrared spectroscopy in the detection of regional tissue oxygenation during hypoxic events in preterm infants undergoing critical care, Ped. Crit. Care Med. 7 (2006), 449-454. 
[104] R.N. Pittman, Oxygen supply to contracting skeletal muscle at the microcirculatory level: diffusion vs. convection, Acta Physiol. Scand. 168 (2000), 593-602.

[105] V. Quaresima, T. Komiyama and M. Ferrari, Differences in oxygen re-saturation of thigh and calf muscles after two treadmill stress tests, Comparative Biochemistry and Physiology Part A: Molecular \& Integrative Physiology 132(1) (2002), 67-73.

[106] V. Quaresima, R. Lepanto and M. Ferrari, The use of near infrared spectroscopy in sports medicine, Medicine and Physical Fitness 43(1) (2003), 1-13.

[107] R. Sakakibara, F. Tateno, M. Yano, O. Takahasi, M. Sugiyama, T. Ogata, H. Haruta, M. Kishi, Y. Tsuyusaki, T. Yamamoto, T. Uchiyama, T. Yamanishi and C. Yamaguchi, Imidafenacin on bladder and cognitive function in neurologic OAB patients, Clin. Auton. Res. 23(4) (2013), 189-195.

[108] R. Sakakibara, F. Tateno, M. Yano, O. Takahasi, M. Sugiyama, T. Ogata, M. Kishi, Y. Tsuyusaki, T. Yamamoto, T. Uchiyama, T. Yamanishi and C. Shibata, Tolterodine activates the prefrontal cortex during bladder filling in OAB patients: A real-time NIRS-urodynamics study, Neurourol. Urodyn. 33(7) (2014), 1110-1115.

[109] R. Sakakibara, K. Tsunoyama, O. Takahasi, M. Sugiyama, M. Kishi, E. Ogawa, T. Uchiyama, T. Yamamoto, T. Yamanishi, Y. Awa and C. Yamaguchi, Real-time measurement of oxyhemoglobin concentration changes in the frontal micturition area: an fNIRS study, Neurourol. Urodyn. 229 (2010), 757-764.

[110] K.P. Sarma, Microangiography of the bladder in health, Br. J. Urol. 53 (1981), 237-240.

[111] S. Segal, Regulation of blood flow in the microcirculation, Microcirculation 12 (2005), 33-45.

[112] B. Shadgan, K. Afshar, L. Stothers and A.J. Macnab, Near-infrared spectroscopy of the bladder: a new technique for studying lower urinary tract function in health and disease, Proc. SPIE 7548 (2010), 754804U-1.

[113] B. Shadgan, M. Fareghi, L. Stothers, A.J. Macnab and A.M. Kajbafzadeh, Diagnosis of testicular torsion using near infrared spectroscopy; a novel diagnostic approach, Can. Urol. Assoc. J. 8(3,4) (2014), E249-E252.

[114] B. Shadgan, A.J. Macnab and L. Stothers, Optical diagnosis of interstitial cystitis/painful bladder syndrome, Proc. SPIE 8565 (2013), 856510.

[115] B. Shadgan, A.J. Macnab, L. Stothers and M. Nigro, Monitoring of lower urinary tract function in patients with spinal cord injury using near infrared spectroscopy, Proc. SPIE 8027 (2012), 802717-1.

[116] B. Shadgan, M. Nigro, A. Macnab, L. Stothers, M. Fareghi, L. Sharifirad and A.M. Kajbafzadeh, Optical diagnosis of lower urinary tract infection: a pilot study in children, Pediatr. Urol. 191(4), Supplement (2014), e75.

[117] B. Shadgan, W.D. Reid, R. Gharakhanlou, L. Stothers and A.J. Macnab, Wireless near-infra-red spectroscopy of skeletal muscle oxygenation and hemodynamics during exercise and ischemia, Spectroscopy 23(5) (2009), 233-241.

[118] B. Shadgan, L. Stothers and A.J. Macnab, A transvaginal probe for near-infrared spectroscopic monitoring of the bladder detrusor muscle and urethral sphincter, Spectroscopy 22(6) (2008), 429-436.

[119] M. Smith and C.E. Elwell, Investigation of in vivo measurement of cerebral cytochrome-c-oxidase redox changes using near-infrared spectroscopy in patients with orthostatic hypotension, Physiol. Meas. 28 (2007), 199-211.

[120] W.F. Stewart, J.B. Van Rooyen, G.W. Cundiff, P. Abrahams, A.R. Herzog, R. Corey, T.L. Hunt and A.J. Wein, Prevalence and burden of overactive bladder in the United States, World J. Urol. 20 (2003), 327-336.

[121] L. Stothers, R. Guevara and A.J. Macnab, Classification of male lower urinary tract symptoms using mathematical modeling and a regression tree algorithm of non-invasive near infrared spectroscopy parameters, Euro. Urol. 57(2) (2010), 327-333.

[122] L. Stothers, A. Macnab and R. Gagnon, Changes in cytochrome C levels in the human bladder during the filling and emptying cycle, J. Urol. 173(Suppl. 4) (2005), 353-354.

[123] L. Stothers and A.J. Macnab, Near-infrared Spectroscopy (NIRS) changes in oxy and deoxyhemoglobin during natural bladder filling and voiding in normal volunteers, J. Urol. 177(Suppl. 4) (2007), 506.

[124] L. Stothers, A. Marques, B. Shadgan and A. Macnab, Near infrared spectroscopy of pelvic floor muscles shows the effect of pelvic floor muscle training in women with stress incontinence, J. Urol. 185(Suppl. 4) (2011), e871.

[125] L. Stothers, B. Shadgan and A.J. Macnab, Urologic applications of infrared spectroscopy (NIRS), Can. J. Urol. 15(6) (2008), 4399-4409.

[126] L. Stothers, B. Shadgan and A.J. Macnab, Near-infrared spectroscopy of the detrusor during urodynamics with simultaneous ultrasound measurements of bladder dimensions and position, Biomedical Spectroscopy and Imaging 1(2) (2012), $137-145$.

[127] S. Suzuki, S. Takasaki, T. Ozaki and Y. Kobayashi, A tissue oxygenation monitor using NIR spatially resolved spectroscopy, Proc. SPIE 3597 (1999), 582-592.

[128] T. Svensson, M. Einarsdóttír, K. Svanberg and S. Andersson-Engels, In vivo optical characterization of human prostate tissue using near-infrared time-resolved spectroscopy, J. Biomed. Opt. 12(1) (2007), 014022.

[129] A.E. Te, D.E. Chung, R.I. Lee and S.A. Kaplan, Near infrared spectroscopy (NIRS) for application in urology, J. Urol. 181(Suppl. 4) (2009), 601-602.

[130] M.C. Van Beekvelt, W.N. Colier, R.A. Wevers and B.G.M. Van Engelen, Performance of near-infrared spectroscopy in measuring local oxygen consumption and blood flow in skeletal muscle, J. Appl. Physiol. 90 (2001), 511-519. 
[131] M.C.P. van Beekvelt, M.S. Borghuis, B.G.M. van Engelen, R.A. Wevers and W.N. Colier, Adipose tissue thickness affects in vivo quantitative near-IR spectroscopy in human skeletal muscle, Clin. Sci. (London) 101(1) (2001), 21-28.

[132] M.C.P. van Beekvelt, K. Orbon, B.G.M. van Engelen, R.A. Wevers and W.N.J.M. Colier, NIR spectroscopic measurement of local muscle metabolism during rhythmic, sustained, and intermittent handgrip exercise, Proc. SPIE 5138 (2003), $35-45$.

[133] M.C.P. van Beekvelt, C.P. van Baziel, G.M. van Engelen, R.A. Wevers and W.N.J.M. Colier, In vivo quantitative nearinfrared spectroscopy in skeletal muscle during incremental isometric handgrip exercise, Clin. Physiol. Fun. Imaging 22(3) (2002), 210-217.

[134] J.P. Van de Merwe, J. Nordling, P. Bouchelouche, K. Bouchelouche, M. Cervigni, L.K. Daha, S. Elneil, M. Fall, G. Hohlbrugger, P. Irwin, S. Mortensen, A. van Ophoven, J.L. Osborne, R. Peeker, B. Richter, C. Riedl, J. Sairanen, M. Tinzl and J.-J. Wyndaele, Diagnostic criteria, classification, and nomenclature for painful bladder syndrome/interstitial cystitis: an ESSIC proposal, Eur. Urol. 53 (2008), 60-67.

[135] P. van de Zee, M. Cope, S.R. Arridge, M. Essenpreis, L.A. Potter, A.D. Edwards, J.S. Wyatt, D.C. McCormick, S.C. Roth, E.O.R. Reynolds and D.T. Delpy, Experimentally measured optical pathlengths for the adult head, calf, and forearm, and the head of the newborn infant as a function of interoptode spacing, Adv. Exper. Med. Biol. 316 (1992), 143-153.

[136] M.C. van der Sluijs, W.N.J.M. Colier, R.J.F. Houston and B. Oesburg, A new and highly sensitive continuous wave near infrared spectrophotometer with multiple detectors, Proc. SPIE 3194 (1977), 63-72.

[137] J.R. Vane, E.E. Anggard and R.M. Botting, Regulatory functions of the vascular endothelium, N. Engl. J. Med. 323 (1990), 27-36.

[138] E.C. Vaux, D.J. Taylor, P. Altmann, B. Rajagopalan, K. Graham, R. Cooper, Y. Bonomo and P. Styles, Effects of carnitine supplementation on muscle metabolism by the use of magnetic resonance spectroscopy and near-infrared spectroscopy in end-stage renal disease, Nephron. Clin. Pract. 97(2) (2004), 41-48.

[139] M. Vij, S. Srikrishna and L. Cardozo, Interstitial cystitis: diagnosis and management, Eur. J. Obstet. Gynecol. Reprod. Biol. 1 (2005), 1-7.

[140] G. Vijaya, G.A. Digesu, A. Erpas, D.C. Panayai, R. Frenando and V. Kjullar, Changes in detrusor muscle oxygenation during detrusor overactivity contractions, Eur. J. Obstet. Gynecol. 163(1) (2012), 104-107.

[141] J.H. Williams, W.H. Turner, G.M. Sainsbury and A.F. Brading, Experimental model of bladder outflow tract obstruction in the guinea pig, Br. J. Urol. Int. 71 (1993), 543-544.

[142] M. Wolf, M. Ferrari and V. Quaresima, Progress of near-infrared spectroscopy and topography for brain and muscle clinical applications, J. Biomed. Optics 12(6) (2007), 062104.

[143] U. Wolf, M. Wolf, J.H. Choi, M. Levi, D. Choudhury, S. Hull, D. Coussirat, L.A. Paunescu, L.P. Safonova, A. Michalos, W.W. Mantulin and E. Graton, Localized irregularities in hemoglobin flow and oxygenation in calf muscle in patients with peripheral vascular disease detected with near-infrared spectrophotometry, J. Vasc. Surg. 37(5) (2003), 1017-1026.

[144] M. Yurt, E. Suer, O. Gulpinar, O. Telli and N. Arikan, Diagnosis of bladder outlet obstruction in men with lower urinary tract symptoms: Comparison of near infrared spectroscopy algorithm and pressure flow studies, Urology 80(1) (2012), $182-186$.

[145] P.I. Zhang, Y. Yang, Z.J. Wu, C.H. Zhang and X.D. Zhang, Diagnosis of bladder outlet obstruction in men using a nearinfrared spectroscopy instrument as the noninvasive monitor for bladder function, Urology 82(5) (2013), 1098-1102. 